\title{
Diagnosis for Nonmagnetic Topological Semimetals in the Absence of Spin-Orbital Coupling
}

\author{
Zhida Song, ${ }^{1,2}$ Tiantian Zhang, ${ }^{1,2}$ and Chen Fang ${ }^{1,3, *}$ \\ ${ }^{1}$ Beijing National Laboratory for Condensed Matter Physics, and Institute of Physics, \\ Chinese Academy of Sciences, Beijing 100190, China \\ ${ }^{2}$ University of Chinese Academy of Sciences, Beijing 100049, China \\ ${ }^{3}$ CAS Center for Excellence in Topological Quantum Computation, Beijing 100190, China
}

(Received 4 January 2018; revised manuscript received 14 July 2018; published 14 September 2018)

\begin{abstract}
Topological semimetals are under intensive theoretical and experimental studies. The first step of these studies is always the theoretical (numerical) predication of one or several candidate materials, based on first-principles numerics. In these calculations, it is crucial that all topological band crossings, including their types and positions in the Brillouin zone, are found. While band crossings along high-symmetry lines, which are routinely scanned in numerics, are simple to locate, the ones at generic momenta are notoriously time-consuming to find and may be easily missed. In this paper, we establish a theoretical scheme of diagnosis for topological semimetals where all band crossings are at generic momenta in systems with time-reversal symmetry and negligible spin-orbital coupling. The scheme uses only the symmetry (inversion and rotation) eigenvalues of the valence bands at high-symmetry points in the Brillouin zone as input and provides the types (lines or points), topological charges, numbers, and configurations of all robust topological band crossings, if any, at generic momenta. The nature of the new diagnosis scheme allows for full automation and parallelization and paves the way to high-throughput numerical predictions of topological semimetals.
\end{abstract}

DOI: 10.1103/PhysRevX.8.031069

Subject Areas: Condensed Matter Physics

\section{INTRODUCTION}

Topologically protected Weyl points [1-3] and nodal lines [4] are being actively searched for and studied in boson bands, such as photons [5,6], phonons [7,8], and magnons [9-13], as well as in electronic bands [14-16]. On one hand, these topological band crossings bring about novel physical properties such as the existence of "arcs" for equal energy contours on the surface $[2,17]$ and the quantum anomalies in the bulk [18]. On the other hand, they are considered the "parent states" of many an interesting topological gapped and gapless state, if certain symmetries are broken by either natural or applied perturbation, such as spin-orbital coupling (SOC) or external strain. For example, a 2D honeycomb lattice hosts two Dirac points, but, when SOC is present, it becomes a topological insulator characterized by the famous Kane-Mele model [19,20]; TaAs is a 3D nodal-line semimetal without considering SOC, but, when it is included (even only perturbatively), the nodal lines break into Weyl points [21,22].

*cfang@iphy.ac.cn

Published by the American Physical Society under the terms of the Creative Commons Attribution 4.0 International license. Further distribution of this work must maintain attribution to the author(s) and the published article's title, journal citation, and DOI.
Some topological band crossings appear at highsymmetry momenta. These include point-type crossings (Weyl and Dirac points) along high-symmetry lines or linetype crossings on high-symmetry planes. The numerical diagnosis of these crossings, that is, predicting their existence, types, and configurations from first principles, is relatively easy, as only a limited number of lines in the band structure should be scanned, a standard protocol integrated in most first-principles implementations nowadays [23]. On the other hand, the diagnosis for the topological band crossings at generic momenta is difficult, because, in principle, the entire Brillouin zone needs to be scanned; and in order to confirm the type of a crossing point, advanced methods such as Wilson loops [24] must be invoked to calculate its topological invariant. This involved process severely slows down the numerical search for more topological semimetals in real materials.

Very recently, several seminal works have established the mathematical relations between the connectivity of bands in a band structure and the irreducible representations of space groups in the valence bands [25-27]. For each space group, a set of equations called the "compatibility relations" are found, which are satisfied if and only if a band structure does not have any (nonaccidental) crossing between the conduction and the valence bands along all high-symmetry lines in the Brillouin zone. If any one of the compatibility relations is violated, the system must be a 
topological semimetal with a band crossing at highsymmetry momenta. On the other hand, if all equations are met, there are two possibilities: The band structure either is fully gapped at all momenta (insulator) or has band crossings at generic momenta. References $[25,26]$ further study the band structures of atomic insulators (or their superpositions), which form a linear space spanned by basis vectors called the "elementary band representations." These authors point out that, if a band structure satisfies all compatibility relations but cannot linear-decompose into elementary band representations with integer coefficients, it must be either a topological gapped state or a topological semimetal. Both the compatibility relations and the explicit expressions of elementary band representations are now fully available online at BILBAO thanks to the effort of Refs. [26,28,29].

Po, Vishwanath, and Watanabe (PVW) [25] show that, given that all compatibility relations are satisfied, the numbers of the appearance of each irreducible representation at all high-symmetry momenta in the valence bands (defined as "symmetry data" for short) can be converted into a very small set containing at most four $\mathbb{Z}_{n}$ numbers with $n=2,4$ for the orthogonal Hamiltonians (time reversal without SOC) and $n=2,3,4,6,8,12$ for the symplectic ones (time reversal with SOC). These numbers are termed "symmetry-based indicators" (indicator for short). Indicators are indicative of nontrivial topology in a band structure such that any system with nonzero indicators cannot be an atomic insulator but can be only either a topologically gapped state or a topological semimetal. Mark here that, in the latter possibility, all band crossings must appear at generic momenta, because the compatibility relations are given as satisfied. More importantly, any system having zero indicators has symmetry data that cannot be distinguished from that of an atomic insulator $[25,26]$. This property means that all information on band topology contained in symmetry data can be extracted from indicators alone. However, the mathematical framework used by PVW does not tell us to what topological states each nonzero set of indicators corresponds: Is the material an insulator or a semimetal? If an insulator, what are the topological invariants? If a semimetal, what are the types and configurations of the band crossings? Also, Ref. [25] contains only the groups formed by indicators (or the indicator groups) without giving the explicit isomorphism between the symmetry eigenvalues and the generators of that group.

This paper is tasked with answering these questions in systems with time reversal and negligible SOC, filling the gaps between symmetry data and band topology by (i) giving each generator of each indicator group an explicit formula in terms of symmetry eigenvalues of valence bands at high-symmetry momenta and (ii) "translating" each nonzero set of indicators into a set of well-defined topological states. The main results can be parsed into two statements:
(1) All nonzero sets of indicators in all space groups necessarily correspond to topological semimetals but not insulators.

(2) The types, numbers, and configurations of the band crossings can be partly predicted by the indicators. Both results are obtained by examining each indicator in every space group, where we first find the explicit formula for that indicator, prove that a band crossing must exist whenever it becomes nonzero, determine the type of the crossing, and finally give a possible configuration. In centrosymmetric space groups, all these topological semimetals are nodal-line semimetals, where the nodal lines are away from high-symmetry points, lines, or planes. Particularly, we find that, for many space groups, there is always one set of indicators that corresponds to nodal-line topological semimetals where nodal loops have nontrivial $\mathbb{Z}_{2}$-monopole charge, a special type of nodal loops that are not predicted in any realistic electronic materials. In noncentrosymmetric space groups, all nonzero sets of indicators correspond to Weyl semimetals, where the Weyl points are away from high-symmetry lines.

In finding the expressions for all indicators, we happily discover, without having such expectations to begin with, that most of them are, in fact, Berry phases along certain loops formed by high-symmetry lines and others the difference of topological charge between two highsymmetry planes. They are all (except two) topological invariants of some submanifolds of the Brillouin zone (BZ), and, if nonzero, they give precise information on the number and positions of band crossings in the submanifold.

\section{PRELIMINARIES: SYMMETRY-BASED INDICATORS}

In this section, we show how the generators of an indicator group are chosen and how the corresponding formulas are derived. In doing this, we first introduce an abstract framework and then give two examples showing the calculations step by step.

\section{A. General theory}

As introduced in the previous section, the symmetry data of a band structure are given by the number of each irreducible representation in the occupied bands. In the following, we denote this number as $n_{\xi_{j}}^{K_{i}}$, where the $K_{i}$ represents the high-symmetry momentum and $\xi_{j}$ represents an irreducible representation at $K_{i}$. Then, the symmetry data of a band structure can be written as a "vector"

$$
\mathbf{n}=\left(n_{\xi_{1}}^{\mathbf{K}_{1}}, n_{\xi_{2}}^{\mathbf{K}_{1}}, \ldots, n_{\xi_{1}}^{\mathbf{K}_{2}}, \ldots\right)^{T}
$$

In principle, $n_{\xi_{i}}^{\mathbf{K}_{j}}$,s should be non-negative integers; however, here we generalize them to arbitrary integers [25]. Since we are interested only in semimetals with crossing 
points at generic momenta, we require $\mathbf{n}$ to satisfy the compatibility relations $[25,26]$, which are a set of constraints such as symmetry eigenvalue conservation along highsymmetry lines, such that the corresponding band structure has no symmetry-protected crossings at high-symmetry momenta. Compatibility relations for all space groups are available online at BILBAO derived in Refs. [26,28,29]. Mathematically, these constraints are described by a set of linear equations, and the compatibility-relation-allowed symmetry data form the solution subspace of these linear equations. Because all entries of $\mathbf{n}$ should be integer, this solution space is an infinite Abelian group, $\mathbb{Z}^{d_{\mathrm{BS}}}$ [27]. In the following, we denote this Abelian group as $\{\mathrm{BS}\}$ and denote its generators as $\mathbf{b}_{i=1 \ldots d_{\mathrm{BS}}}$. Here, $d_{\mathrm{BS}}$ is the rank of $\{\mathrm{BS}\}$.

Another perspective to understand symmetry data is from atomic insulators. Atomic insulators are defined as insulators consisting of uncoupled atoms with closed shells, where, to meet the symmetry, atomic orbitals form the irreducible representations of the corresponding on-site symmetry group. By definition, the generated symmetry data satisfy the compatibility relations and, thus, form a subgroup, denoted as $\{\mathrm{AI}\}$, of $\{\mathrm{BS}\}$. The authors of Ref. [25] prove that $\{\mathrm{AI}\}$ has the same rank as $\{\mathrm{BS}\}$, and, thus, the quotient group $\{\mathrm{BS}\} /\{\mathrm{AI}\}$ is finite. Concretely, for each $\mathbf{b}_{i}$ there exists a minimal integer $\kappa_{i}$ giving $\kappa_{i} \mathbf{b}_{i} \in\{\mathrm{AI}\}$. Therefore, any symmetry data in $\{\mathrm{BS}\}$ can be decomposed into a part belonging to $\{\mathrm{AI}\}$ and a part not belonging to $\{\mathrm{AI}\}$ :

$$
\begin{aligned}
\forall \mathbf{n} & =\sum_{i} c_{i} \mathbf{b}_{i} \in\{\mathrm{BS}\}, \quad \exists \mathbf{n}^{\prime} \in\{\mathrm{AI}\}, \quad \text { such that } \\
\mathbf{n} & =\mathbf{n}^{\prime}+\sum_{i}\left(c_{i} \bmod \kappa_{i}\right) \mathbf{b}_{i},
\end{aligned}
$$

where $\sum_{i}\left(c_{i} \bmod \kappa_{i}\right) \mathbf{b}_{i}$ is the part not belonging to $\{\mathrm{AI}\}$. Such a decomposition implies that the quotient group $\{\mathrm{BS}\} /\{\mathrm{AI}\}$ is $\mathbb{Z}_{\kappa_{1}} \times \mathbb{Z}_{\kappa_{2}} \times \ldots$. The integers $\left(c_{i} \bmod \kappa_{i}\right)$ 's, which identify $\mathbf{n}$ with one element of $\{\mathrm{BS}\} /\{\mathrm{AI}\}$, are defined as the indicators of $\mathbf{n}$, and, hence, the quotient group $\{\mathrm{BS}\} /\{\mathrm{AI}\}$ is also referred to as the indicator group. Band structures having different indicators must be topologically distinct from each other, because their difference, the symmetry data of which does not belong to $\{\mathrm{AI}\}$ according to Eq. (2), cannot be an atomic insulator. Generally, indicators depend on the choice of $\{\mathrm{BS}\}$ generators. Upon a change of the choice of $\{\mathrm{BS}\}$ generators, the values of indicators change, but the indicator group remains the same. More importantly, the redefined indicators with the new $\{\mathrm{BS}\}$ generators are still valid, because distinct indicators still correspond to distinct topologies. In Sec. II B, we take space group 2 as an example to show such a redefinition in more detail. In the rest of this paper, we call the choice of $\{\mathrm{BS}\}$ generators as the convention of indicators. For concreteness, in the following two subsections and Secs. III and IV, we fix the conventions of indicators in all space groups.

The formulas to calculate indicators indeed form a linear mapping from $\{\mathrm{BS}\}$ to the indicator group. Thus, we express the formulas of the $i$ th indicator as $F_{i}(\mathbf{n}) \equiv \mathbf{f}_{i}^{T} \mathbf{n} \bmod \kappa_{i}$, where $\mathbf{f}_{i}$ gives the explicit form of the formulas and satisfies $\mathbf{f}_{i}^{T} \mathbf{b}_{j}=\delta_{i j} \bmod \kappa_{i}$. We emphasize that, even for a fixed indicator convention, the explicit forms of the formulas, i.e., $\mathbf{f}_{i}$ 's, are, in general, not unique due to compatibility relations. Since all symmetry data in $\{\mathrm{BS}\}$ satisfy compatibility relations, which take the form $\mathbf{c}^{T} \mathbf{n}=0$, we can add $\mathbf{c}$ to $\mathbf{f}_{i}$ 's without changing the results, i.e., $F_{i}^{\prime}(\mathbf{n}) \equiv\left(\mathbf{f}_{i}+\mathbf{c}\right)^{T} \mathbf{n} \bmod \kappa_{i}=F_{i}(\mathbf{n})$. A general strategy to get one of the many equivalent explicit forms is to solve the left inverse of the nonsquare matrix $B=\left(\mathbf{b}_{1}, \mathbf{b}_{2}, \ldots\right), B_{\text {left }}^{-1}$, such that $B_{\text {left }}^{-1} B=\mathbb{I}$. Then, the $\mathbf{f}_{i}$ vector is given by the $i$ th column of $B_{\text {left }}^{-1 T}$.

In order to simplify finding explicit indicator formulas and get a consistent understanding of indicators in all space groups, here we present a method to get indicators of a space group from indicators of its subgroup. We denote the group and its subgroup as $G$ and $H$, respectively. We first notice that the indicators of $H$, which are expressed as functions of the symmetry data in $H$, are also well defined for that in $G$, as each irreducible representation of $G$ necessarily reduces into one or multiple irreducible representations in $H$. Then, if two band structures in $G$ have distinct indicators of $H$, which imply topological distinction, they must have distinct indicators of $G$, because, by definition, topologies that can be distinguished from symmetry data must have distinct indicators. In other words, any two distinct realizable indicators of $H$ in $G$ correspond to two distinct indicators of $G$. Here, by realizable indicators of $H$ in $G$, we mean that there is at least one symmetry data of $G$ that (i) satisfies all compatibility relations of $G$ and (ii) has such a set of indicators of $H$. If the realizable indicators of $H$ happen to form the indicator group of $G$, we say that the indicator group of $G$ is completely induced from $H$; otherwise, we say that the indicator group of $G$ is partly induced from $H$. In the latter case, new formulas for the remaining indicators must be defined. In Sec. IIC, we give a concrete example for indicator induction.

\section{B. Space group 2: The simplest example}

Space group 2 is generated by three lattice translations along three lattice vectors $\mathbf{a}_{1,2,3}$ and inversion symmetry [30]. The eight high-symmetry momenta are the eight time-reversal invariant momenta (TRIMs), i.e., $\left(k_{1}, k_{2}, k_{3}\right)$ with $k_{1,2,3}=0, \pi$. [Hereafter, we use $\left(k_{1}, k_{2}, k_{3}\right)$ to denote momentum in reciprocal lattices, i.e., $\mathbf{k}=k_{1} \mathbf{g}_{1}+k_{2} \mathbf{g}_{2}+$ $k_{3} \mathbf{g}_{3}$, where $\mathbf{g}_{i=1,2,3}$ are the reciprocal bases, and use $\left(k_{x}, k_{y}, k_{z}\right)$ to denote momentum in Cartesian coordinates. Definitions of the reciprocal lattices in all space groups can be found on the BILBAO Web site [31], and the parts 
used in this paper are tabulated in Table III.] On each of them, there are two kinds of one-dimensional irreducible representations: the even under inversion and the odd under inversion. Thus, the symmetry data take the form $\mathbf{n}=\left(n_{+}^{\mathbf{K}_{1}}, n_{-}^{\mathbf{K}_{1}}, \ldots, n_{+}^{\mathbf{K}_{8}}, n_{-}^{\mathbf{K}_{8}}\right)^{T}$, where $n_{ \pm}^{\mathbf{K}_{i}}$ represents the number of occupied states having the inversion eigenvalue \pm 1 at the $i$ th TRIM. The only compatibility relation in space group 2 is to require the same occupation numbers at different TRIMs, i.e.,

$$
n_{+}^{\mathbf{K}_{i}}+n_{-}^{\mathbf{K}_{i}}-n_{+}^{\mathbf{K}_{j}}-n_{-}^{\mathbf{K}_{j}}=0 \quad(i, j=1, \ldots, 8) .
$$

The solution spaces of these equations are easy to get, and we list the nine generators in Table I.

According to Ref. [26], to generate $\{\mathrm{AI}\}$, only the orbitals at the "maximal Wyckoff positions" need to be considered. Here, maximal Wyckoff position refers to the

TABLE I. The generators of $\{\mathrm{BS}\}$ and $\{\mathrm{AI}\}$ of space group 2 . Here, $\mathbf{e}_{ \pm}^{\mathbf{K}}$ represents the basis where all entries are zero except $n_{ \pm}^{\mathbf{K}}=1, \kappa_{i}$ represents the order of $\mathbf{b}_{i}$, i.e., the minimal positive integer making $\kappa_{i} \mathbf{b}_{i} \in\{\mathrm{AI}\}$, and the symbol $G_{ \pm}^{w}$ in parentheses represents that the corresponding atomic symmetry data are induced from the orbital having the $P$ eigenvalue \pm 1 at the Wyckoff position $w$. The notations for momenta and positions are defined as $\Gamma(000), R(\pi \pi \pi), T(0 \pi \pi), U(\pi 0 \pi), V(\pi \pi 0), X(\pi 00)$, $Y(0 \pi 0), Z(00 \pi)$ and $1 a(000), 1 b\left(00 \frac{1}{2}\right), 1 c\left(0 \frac{1}{2} 0\right), 1 d\left(\frac{1}{2} 00\right)$, $1 e\left(\frac{1}{2} \frac{1}{2} 0\right), 1 f\left(\frac{1}{2} 0 \frac{1}{2}\right), 1 g\left(0 \frac{1}{2} \frac{1}{2}\right), 1 h\left(\frac{1}{2} \frac{1}{2} \frac{1}{2}\right)$, respectively.

Generators of $\{\mathrm{BS}\}$

\begin{tabular}{lll}
\hline Basis & \multicolumn{1}{c}{ Symmetry data } & $\kappa_{i}$ \\
\hline $\mathbf{b}_{1}$ & $\mathbf{e}_{-}^{\Gamma}+\mathbf{e}_{-}^{R}+\mathbf{e}_{+}^{T}+\mathbf{e}_{+}^{U}+\mathbf{e}_{-}^{V}+\mathbf{e}_{+}^{X}+\mathbf{e}_{+}^{Y}+\mathbf{e}_{-}^{Z}$ & 1 \\
$\mathbf{b}_{2}$ & $\mathbf{e}_{-}^{\Gamma}+\mathbf{e}_{-}^{R}+\mathbf{e}_{-}^{T}+\mathbf{e}_{+}^{U}+\mathbf{e}_{+}^{V}+\mathbf{e}_{-}^{X}+\mathbf{e}_{+}^{Y}+\mathbf{e}_{+}^{Z}$ & 1 \\
$\mathbf{b}_{3}$ & $\mathbf{e}_{+}^{T}-\mathbf{e}_{-}^{T}-\mathbf{e}_{+}^{U}+\mathbf{e}_{-}^{U}+\mathbf{e}_{+}^{X}-\mathbf{e}_{-}^{X}-\mathbf{e}_{+}^{Y}+\mathbf{e}_{-}^{Y}$ & 1 \\
$\mathbf{b}_{4}$ & $-\mathbf{e}_{+}^{R}+\mathbf{e}_{-}^{R}+\mathbf{e}_{+}^{U}-\mathbf{e}_{-}^{U}+\mathbf{e}_{+}^{V}-\mathbf{e}_{-}^{V}-\mathbf{e}_{+}^{X}+\mathbf{e}_{-}^{X}$ & 1 \\
$\mathbf{b}_{5}$ & $-\mathbf{e}_{+}^{\Gamma}+\mathbf{e}_{-}^{\Gamma}-\mathbf{e}_{+}^{T}+\mathbf{e}_{-}^{T}-\mathbf{e}_{+}^{U}+\mathbf{e}_{-}^{U}-\mathbf{e}_{+}^{V}+\mathbf{e}_{-}^{V}$ & 1 \\
$\mathbf{b}_{6}$ & $-\mathbf{e}_{+}^{\Gamma}+\mathbf{e}_{-}^{\Gamma}+\mathbf{e}_{+}^{X}-\mathbf{e}_{-}^{X}$ & 2 \\
$\mathbf{b}_{7}$ & $-\mathbf{e}_{+}^{\Gamma}+\mathbf{e}_{-}^{\Gamma}+\mathbf{e}_{+}^{Y}-\mathbf{e}_{-}^{Y}$ & 2 \\
$\mathbf{b}_{8}$ & $-\mathbf{e}_{+}^{\Gamma}+\mathbf{e}_{-}^{\Gamma}+\mathbf{e}_{+}^{Z}-\mathbf{e}_{-}^{Z}$ & 4 \\
$\mathbf{b}_{9}$ & $-\mathbf{e}_{+}^{\Gamma}+\mathbf{e}_{-}^{\Gamma}$ &
\end{tabular}

Generators of $\{\mathrm{AI}\}$

\begin{tabular}{ll}
\hline Basis & \multicolumn{1}{c}{ Symmetry data } \\
\hline $\mathbf{a}_{1}\left(G_{+}^{1 a}\right)$ & $\mathbf{e}_{+}^{\Gamma}+\mathbf{e}_{+}^{R}+\mathbf{e}_{+}^{T}+\mathbf{e}_{+}^{U}+\mathbf{e}_{+}^{V}+\mathbf{e}_{+}^{X}+\mathbf{e}_{+}^{Y}+\mathbf{e}_{+}^{Z}$ \\
$\mathbf{a}_{2}\left(G_{-}^{1 a}\right)$ & $\mathbf{e}_{-}^{\Gamma}+\mathbf{e}_{-}^{R}+\mathbf{e}_{-}^{T}+\mathbf{e}_{-}^{U}+\mathbf{e}_{-}^{V}+\mathbf{e}_{-}^{X}+\mathbf{e}_{-}^{Y}+\mathbf{e}_{-}^{Z}$ \\
$\mathbf{a}_{3}\left(G_{+}^{1 b}\right)$ & $\mathbf{e}_{+}^{\Gamma}+\mathbf{e}_{-}^{R}+\mathbf{e}_{-}^{T}+\mathbf{e}_{-}^{U}+\mathbf{e}_{+}^{V}+\mathbf{e}_{+}^{X}+\mathbf{e}_{+}^{Y}+\mathbf{e}_{-}^{Z}$ \\
$\mathbf{a}_{4}\left(G_{+}^{1 c}\right)$ & $\mathbf{e}_{+}^{\Gamma}+\mathbf{e}_{-}^{R}+\mathbf{e}_{-}^{T}+\mathbf{e}_{+}^{U}+\mathbf{e}_{-}^{V}+\mathbf{e}_{+}^{X}+\mathbf{e}_{-}^{Y}+\mathbf{e}_{+}^{Z}$ \\
$\mathbf{a}_{5}\left(G_{+}^{1 d}\right)$ & $\mathbf{e}_{+}^{\Gamma}+\mathbf{e}_{-}^{R}+\mathbf{e}_{+}^{T}+\mathbf{e}_{-}^{U}+\mathbf{e}_{-}^{V}+\mathbf{e}_{-}^{X}+\mathbf{e}_{+}^{Y}+\mathbf{e}_{+}^{Z}$ \\
$\mathbf{a}_{6}\left(G_{+}^{1 e}\right)$ & $\mathbf{e}_{+}^{\Gamma}+\mathbf{e}_{+}^{R}+\mathbf{e}_{-}^{T}+\mathbf{e}_{-}^{U}+\mathbf{e}_{+}^{V}+\mathbf{e}_{-}^{X}+\mathbf{e}_{-}^{Y}+\mathbf{e}_{+}^{Z}$ \\
$\mathbf{a}_{7}\left(G_{+}^{1 f}\right)$ & $\mathbf{e}_{+}^{\Gamma}+\mathbf{e}_{+}^{R}+\mathbf{e}_{-}^{T}+\mathbf{e}_{+}^{U}+\mathbf{e}_{-}^{V}+\mathbf{e}_{-}^{X}+\mathbf{e}_{+}^{Y}+\mathbf{e}_{-}^{Z}$ \\
$\mathbf{a}_{8}\left(G_{+}^{1 g}\right)$ & $\mathbf{e}_{+}^{\Gamma}+\mathbf{e}_{+}^{R}+\mathbf{e}_{+}^{T}+\mathbf{e}_{-}^{U}+\mathbf{e}_{-}^{V}+\mathbf{e}_{+}^{X}+\mathbf{e}_{-}^{Y}+\mathbf{e}_{-}^{Z}$ \\
$\mathbf{a}_{9}\left(G_{+}^{1 h}\right)$ & $\mathbf{e}_{+}^{\Gamma}+\mathbf{e}_{-}^{R}+\mathbf{e}_{+}^{T}+\mathbf{e}_{+}^{U}+\mathbf{e}_{+}^{V}+\mathbf{e}_{X}^{-}+\mathbf{e}_{Y}^{-}+\mathbf{e}_{Z}^{-}$ \\
\hline \hline
\end{tabular}

position whose on-site symmetry group is a maximal finite subgroup of the space group. For space group 2, there are only eight such positions, i.e., $\left(x_{1}, x_{2}, x_{3}\right)$ with $x_{1,2,3}=0, \frac{1}{2}$. Since all of these positions are inversion invariant (modulo a lattice), on each position we have only two kinds of orbitals: the even one and the odd one. We denote the atomic orbital with parity $\chi= \pm 1$ at the inversion-invariant position $\mathbf{t}$ in the lattice $\mathbf{R}$ as $\langle\chi \mathbf{t}+\mathbf{R}\rangle$ and define the corresponding Blöch wave function as $\left|\phi_{\chi \mathbf{t}}(\mathbf{k})\right\rangle=$ $(1 / \sqrt{N}) \sum_{\mathbf{R}} e^{i(\mathbf{t}+\mathbf{R}) \cdot \mathbf{k}}|\chi \mathbf{t}+\mathbf{R}\rangle$. Under inversion operation $P$, the atomic orbital first gets an inversion eigenvalue and then moves to the inverted position, i.e., $\hat{P}|\chi \mathbf{t}+\mathbf{R}\rangle=$ $\chi|\chi-\mathbf{t}-\mathbf{R}\rangle$. (We use unhatted symbols for the symmetries themselves and hatted ones for the corresponding operators.) Using this property, it is direct to obtain the inversion eigenvalues of $\left|\phi_{\chi \mathbf{t}}(\mathbf{k})\right\rangle$ for $\mathbf{k} \in$ TRIMs. By this method, we generate 16 symmetry data (two orbitals for each position and eight positions in total) and find that only nine of them are linearly independent, which is consistent with the statement that $\{\mathrm{AI}\}$ has the same rank as $\{\mathrm{BS}\}$. We choose the nine listed in Table I as the generators of $\{\mathrm{AI}\}$. A crucial observation then follows: $\mathbf{b}_{i=1 \ldots 5}$ can be generated from $\mathbf{a}_{i}$ 's, for example, $\mathbf{b}_{1}=\mathbf{a}_{1}+\mathbf{a}_{2}-\mathbf{a}_{6}$, whereas $\mathbf{b}_{i=6,7,8,9}$ cannot. Nevertheless, $2 \mathbf{b}_{6}, 2 \mathbf{b}_{7}, 2 \mathbf{b}_{8}$, and $4 \mathbf{b}_{9}$ can be generated from $\mathbf{a}_{i}$ 's, for example, $4 \mathbf{b}_{9}=$ $3 \mathbf{a}_{1}+4 \mathbf{a}_{2}-\sum_{i=3}^{9} \mathbf{a}_{i}$. Therefore, we have $\kappa_{i=1 \ldots 5}=1$, $\kappa_{i=6,7,8}=2$, and $\kappa_{i=9}=4$, and the indicator group $\{\mathrm{BS}\} /\{\mathrm{AI}\}$ is $\mathbb{Z}_{2} \times \mathbb{Z}_{2} \times \mathbb{Z}_{2} \times \mathbb{Z}_{4}$.

By counting the numbers of even and odd states at particular TRIMs, we find that the following formulas give a successful mapping from $\{$ BS $\}$ to $\mathbb{Z}_{2} \times \mathbb{Z}_{2} \times \mathbb{Z}_{2} \times \mathbb{Z}_{4}$ :

$$
\begin{aligned}
z_{2,1} & \equiv \sum_{\substack{\mathbf{K} \in \mathrm{TRIM} \\
\text { at }\left\{k_{1}=\pi\right\}}} \frac{N_{-}(\mathbf{K})-N_{+}(\mathbf{K})}{2} \bmod 2, \\
z_{2,2} & \equiv \sum_{\substack{\mathbf{K} \in \operatorname{TRIM} \\
\text { at }\left\{k 2_{2}=\pi\right\}}} \frac{N_{-}(\mathbf{K})-N_{+}(\mathbf{K})}{2} \bmod 2, \\
z_{2,3} & \equiv \sum_{\substack{\mathbf{K} \in \mathrm{TRIM} \\
\text { at }\left\{k_{3}=\pi\right\}}} \frac{N_{-}(\mathbf{K})-N_{+}(\mathbf{K})}{2} \bmod 2, \\
z_{4} & \equiv \sum_{\mathbf{K} \in \mathrm{TRIM}} \frac{N_{-}(\mathbf{K})-N_{+}(\mathbf{K})}{2} \bmod 4,
\end{aligned}
$$

where $N_{ \pm}(\mathbf{K})$ is the number of valence bands having positive (negative) parity. Here, we use the notation $N_{ \pm}(\mathbf{K})$ instead of $n_{ \pm}^{\mathbf{K}}$ to emphasize that these equations are applicable for all centrosymmetric space groups, where $N_{ \pm}(\mathbf{K})$ is obtained from more general symmetry data. For a general centrosymmetric space group, one ignores all symmetries but inversion and translation and calculates $N_{ \pm}(\mathbf{K})$ as if it were a symmetry data of space group 2 . As shown in Sec. III, most indicators in centrosymmetric space groups are indeed induced from the above four equations. 
As mentioned in Sec. II A, the indicators are, in general, convention dependent due to the many choices of $\{\mathrm{BS}\}$ generators. Here, we take the $z_{4}$ indicator as an example to show the convention dependence and discuss the physical interpretation of this convention dependence. Below, we proceed with another choice of $\mathbf{b}_{8}$, but the discussion applies for all $\{\mathrm{BS}\}$ generators. Upon redefining the eighth generator as $\mathbf{b}_{8}-2 \mathbf{b}_{9}$, which leaves the order $\kappa_{8}=2$ invariant, the $z_{2, i=1,2,3}$ indicators remain invariant, whereas the $z_{4}$ indicator changes to $z_{4}+2 z_{2,3} \bmod 4$. Specifically, upon the redefining, the indicator set (0011) interchanges with (0013), and the indicator set (0010) interchanges with (0012). Then, we find that, from the physical point of view, redefining indicators in this way corresponds to a different choice of inversion center from $(000)$ to $\left(00 \frac{1}{2}\right)$. Choosing a different inversion center redefines the inversion operation as $\{\overline{1} \mid 001\}$, i.e., inversion centered at (000) followed by a translation (001). (Following BILBAO [30], we use $\{p \mid \mathbf{t}\}$ to represent the space-group operation composed of point-group operation $p$ followed by translation t.) The translation (001) leads to additional "-" signs in the inversion eigenvalues at the $k_{3}=\pi$ momenta such that the generator $\mathbf{b}_{8}=-\mathbf{e}_{+}^{\Gamma}+\mathbf{e}_{-}^{\Gamma}+\mathbf{e}_{+}^{Z}-\mathbf{e}_{-}^{Z}$, which has indicator set (0010) due to Eq. (4), changes to $\mathbf{b}_{8}=-\mathbf{e}_{+}^{\Gamma}+\mathbf{b}_{8}+\mathbf{e}_{-}^{\Gamma}+\mathbf{e}_{-}^{Z}-\mathbf{e}_{+}^{Z}$, which has the indicator set (0012) due to Eq. (4). (See Table I for definitions of the notation $\mathbf{e}_{ \pm}^{\mathbf{K}}$.) Generalizing the discussion for changing the inversion center from $(000)$ to $[(i / 2)(j / 2)(k / 2)](i, j$, $k=0,1)$, one can easily find that the $z_{2, i=1,2,3}$ indicators remain invariant, whereas the $z_{4}$ indicator changes to $z_{4}+2 i z_{2,1}+2 j z_{2,2}+2 k z_{2,3} \bmod 4$. It should be noticed that, whichever inversion center is chosen, the parity of $z_{4}$ remains the same, i.e., convention independent. In fact, odd $z_{4}$ corresponds to an odd number of nodal loops centering at TRIMs, as discussed in Sec. III.

\section{Space group 10: Example showing indicator induction and more}

Space group 10 is generated by three lattice translations along $\mathbf{a}_{1,2,3}$, an inversion $P$, and a rotation $C_{2}$, wherein the rotation axis passes through the inversion center and is parallel to $\mathbf{a}_{2}$ [30]. In the absence of SOC, these operators satisfy $\hat{P}^{2}=\hat{C}_{2}^{2}=1$ and $\left[\hat{P}, \hat{C}_{2}\right]=0$. There are two types of high-symmetry momenta: (i) the eight TRIMs, which are invariant under both $P$ and the $C_{2}$, and (ii) the four highsymmetry lines $\left(k_{1}, k_{2}, k_{3}\right)\left(k_{1}, k_{3}=0, \pi, k_{2} \neq 0, \pi\right)$, which are invariant under only $C_{2}$. The TRIMs have four onedimensional irreducible representations, which have the $C_{2}$ and $P$ eigenvalues $(1,1),(1,-1),(-1,1)$, and $(-1,-1)$, respectively. Thus, the corresponding entries in symmetry data are given by $n_{ \pm, \pm}^{\mathbf{K}}$, representing the number of states having the $C_{2}$ and $P$ eigenvalues $\pm 1, \pm 1$ at $\mathbf{K}$. On the other hand, the $C_{2}$-invariant lines have only two kinds of onedimensional irreducible representations, i.e., the one having the $C_{2}$ eigenvalue 1 and the one having the $C_{2}$ eigenvalue -1 . Thus, the corresponding entries in symmetry data are given by $n_{ \pm}^{\mathbf{K}}$, where \pm represents the rotation eigenvalue \pm 1 .

Now, we turn to the compatibility relations. The first kind of compatibility relation simply requires the same occupation numbers at high-symmetry momenta. The second kind requires that the $C_{2}$ eigenvalues of the occupied bands remain invariant along the $C_{2}$-invariant lines. On one hand, the second kind of relation makes $n_{ \pm}^{\mathbf{K}}(\mathbf{K} \notin \mathrm{TRIM})$ a constant along a $C_{2}$-invariant line. On the other hand, viewing TRIMs as particular points in the $C_{2}$-invariant lines, these relations require $n_{ \pm,+}^{\mathbf{K}}+n_{ \pm,-}^{\mathbf{K}}=$ $n_{ \pm}^{\mathbf{K}+\left(0, k_{2}, 0\right)}$ for $\mathbf{K} \in$ TRIM and $k_{2} \neq 0, \pi$, implying that the symmetry data on the $C_{2}$-invariant lines are completely determined by the symmetry data on the TRIMs. Therefore, in the following we keep only the symmetry data on the eight TRIMs and denote the eight TRIMs as $\mathbf{K}_{i=1 \ldots .}$. The two kinds of compatibility relation are then given by

$$
\sum_{\zeta= \pm 1, \chi= \pm 1} n_{\zeta, \chi}^{\mathbf{K}_{i}}-n_{\zeta, \chi}^{\mathbf{K}_{j}}=0 \quad(i, j=1 \ldots 8)
$$

and

$$
\sum_{\chi= \pm 1} n_{1, \chi}^{\mathbf{K}_{i}}-n_{1, \chi}^{\mathbf{K}_{i}+(0, \pi, 0)}=0 \quad(i=1 \ldots 8)
$$

respectively. We tabulate the 15 generators of the solutions in Table II.

Generators of $\{\mathrm{AI}\}$ can be calculated by the same method described in Sec. II B. Space group 10 has eight maximal Wyckoff positions [26], i.e., $\left(x_{1}, x_{2}, x_{3}\right)\left(x_{1,2,3}=0, \frac{1}{2}\right)$, each of which has four kinds of atomic orbitals with the $C_{2}$ and $P$ eigenvalues $(1,1),(1,-1),(-1,1)$, and $(-1,-1)$, respectively. By a direct Fourier transformation of these atomic orbitals, we get the 15 independent atomic symmetry data, which are chosen as generators of $\{\mathrm{AI}\}$, shown in Table II. Then, it follows that $\mathbf{b}_{i=1 \ldots 12} \in\{\mathrm{AI}\}$ and $\mathbf{b}_{i=13,14,15} \notin\{\mathrm{AI}\}$, whereas $2 \mathbf{b}_{i=13,14,15} \in\{\mathrm{AI}\}$, implying that the indicator group is $\mathbb{Z}_{2} \times \mathbb{Z}_{2} \times \mathbb{Z}_{2}$.

To find explicit formulas for the indicators, we first notice that group 2 is a subgroup of group 10, and, thus, we can induce the indicator formulas from group 2 by the method described in Sec. II A. Substituting $\mathbf{b}_{i}$ 's into Eq. (4), we find that the group 2 indicator set $\left(z_{2,1} z_{2,2} z_{2,3} z_{4}\right)$ of the three nontrivial generators, $\mathbf{b}_{13,14,15}$, is $(0100)$, (0100), and (0002), respectively, generating a $\mathbb{Z}_{2} \times \mathbb{Z}_{2}$ group. Therefore, two of the three $\mathbb{Z}_{2}$ indicators are induced from $z_{2,2}$ and $z_{4}$ of space group 2 , while the left $\mathbb{Z}_{2}$ indicator is not induced from group 2. Since $z_{4}=1,3$ are no more realizable in space group $10, z_{4}$ reduce to a $\mathbb{Z}_{2}$ number, and we introduce $z_{2}^{\prime}=z_{4} / 2$ to represent this $\mathbb{Z}_{2}$ number. To find the left indicators, we notice that states in 
TABLE II. The generators of $\{\mathrm{BS}\}$ and $\{\mathrm{AI}\}$ of the space group 10. Here, $\mathbf{e}_{ \pm, \pm}^{\mathbf{K}}$ represents the basis where all entries are zero except $n_{ \pm, \pm}^{\mathbf{K}}=1, \kappa_{i}$ represents the order of $\mathbf{b}_{i}$, i.e., the minimal positive integer making $\kappa_{i} \mathbf{b}_{i} \in\{\mathrm{AI}\}$, and the symbol $G_{ \pm, \pm}^{w}$ in parentheses represents that the corresponding atomic symmetry data are induced from the orbital having the $C_{2}$ and $P$ eigenvalues $\pm 1, \pm 1$ at the Wyckoff position $w$. The notations for momenta and positions are defined as $A(\pi 0 \pi), B(00 \pi), C(\pi \pi 0), D(0 \pi \pi), E(\pi \pi \pi), \Gamma(000)$, $Y(\pi 00), Z(0 \pi 0)$ and $1 a(000), 1 b\left(0 \frac{1}{2} 0\right), 1 c\left(00 \frac{1}{2}\right), 1 d\left(\frac{1}{2} 00\right), 1 e\left(\frac{1}{2} \frac{1}{2} 0\right), 1 f\left(0 \frac{1}{2} \frac{1}{2}\right), 1 g\left(\frac{1}{2} 0 \frac{1}{2}\right), 1 h\left(\frac{1}{2} \frac{1}{2} \frac{1}{2}\right)$, respectively.

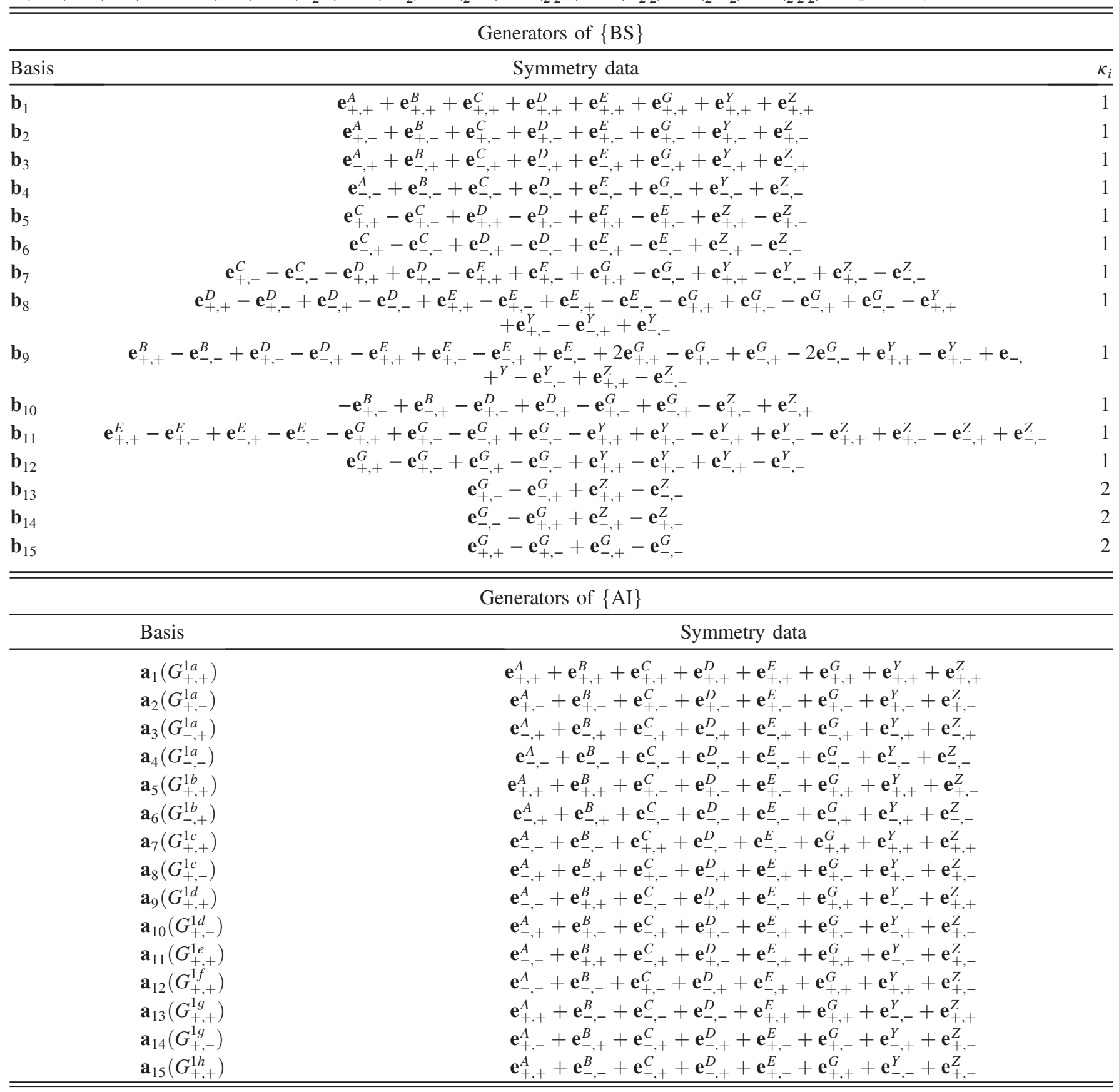

the $k_{2}=\pi$ plane can be divided into two sectors due to their mirror $\left(M=P C_{2}\right)$ eigenvalues, and, thus, the parities in this plane can be counted for the two sectors separately as

$$
z_{2}^{( \pm)} \equiv \sum_{\substack{\mathbf{K} \in \operatorname{TR} I M \\ \text { at }\left\{k_{2}=\pi\right\}}} \frac{N_{-}^{( \pm)}(\mathbf{K})-N_{+}^{( \pm)}(\mathbf{K})}{2} \bmod 2,
$$

where $N_{+}^{( \pm)}(\mathbf{K})$ is the number of states having parity +1 in the $M= \pm 1$ sector and $N_{-}^{( \pm)}(\mathbf{K})$ is the number of states having parity -1 in the $M= \pm 1$ sector. Apparently, the sum of $z_{2}^{(+)}$and $z_{2}^{(-)}$just gives $z_{2,2}(\bmod 2)$, whereas $z_{2}^{(+)}$ and $z_{2}^{(-)}$themselves are new indicators, either of which realizes a successful mapping from $\{\mathrm{BS}\}$ to the missing $\mathbb{Z}_{2}$ 
indicator. Therefore, we choose the independent indicators of space group 10 as $z_{2}^{(+)}, z_{2}^{(-)}$, and $z_{2}^{\prime}$ such that the nontrivial generators $\mathbf{b}_{13,14,15}$ have the indicators (100), (010), and (001), respectively.

\section{CENTROSYMMETRIC SPACE GROUPS}

In the absence of spin-orbital coupling, the time-reversal operator $\hat{T}$ satisfies $\hat{T}^{2}=+1$. Hamiltonians having the $\hat{T}^{2}=+1$ symmetry belong to the orthogonal WignerDyson class [32], also known as class AI in the new Altland-Zirnbauer system [33]. When inversion symmetry $\hat{P}$ is also present, the composite symmetry $P T$ satisfies $(\hat{P} \hat{T})^{2}=+1$. In the presence of $P T$, generic band crossings are nodal lines, which are robust against symmetric perturbations, due to the $\pi$-Berry phase associated with any loop that links with a nodal line [34,35].

First, we introduce a lemma in a 2D system with $P$ and $T$, which later we see has quite a few useful variations. Consider a closed path in the 2D BZ that encircles exact one-half of the BZ such that the inside and the outside of the loop are mapped to each other under inversion [see Fig. 1(a)]. Following Ref. [36], one can prove that the Berry phase of the loop is given by the following.

Lemma.-

$$
e^{i \Phi_{B}}=\prod_{n \in \mathrm{occ}, K \in \mathrm{TRIM}} \chi_{n}(K),
$$

where occ represents the set of valence bands and $\chi_{n}(K)$ the inversion eigenvalue of the $n$th band at $K$. When the product of inversion eigenvalues at TRIM of all valence bands is -1 , the Berry phase is $\pi$, so that there must be one (or an odd number of) Dirac point inside the area enclosed by the loop. Suppose the Dirac point has momenta $\mathbf{k}_{0}$; then, due to time reversal, there is another at $-\mathbf{k}_{0}$, so that there are at least two Dirac points in the 2D BZ.

This lemma helps diagnose the presence and the configuration of nodal loops in the BZ. In a 3D system having $P$ and $T$, there are eight TRIMs that are invariant under parity. The above lemma applies to any $2 \mathrm{D}$ slice in the $3 \mathrm{D}$
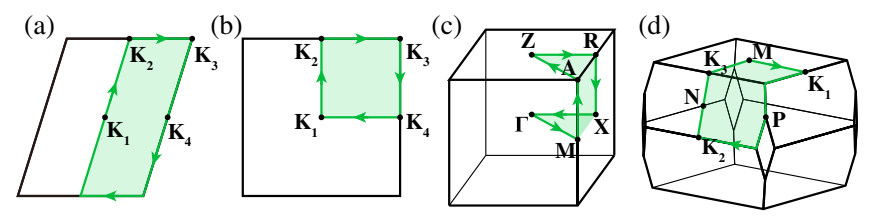

FIG. 1. The loops on which Berry phases are considered in this paper. The plane in (a) can be any 2D submanifold of the 3D BZ in a system having inversion and the plane in (a) and (b) can be any $k$ slice in a system having a twofold or fourfold axis. (a) A loop enclosing half of the BZ, passing four TRIMs on its way, denoted by $\mathbf{K}_{1,2,3,4}$. (b) A loop enclosing a quarter of the BZ, passing all four TRIMs. (c) A loop particularly considered in a simple tetragonal lattice for space group 130.
Brillouin zone as long as the slice is invariant under inversion. It is straightforward to confirm that any such 2D slice passes exactly four out of the eight TRIMs, and, if for that slice $\Phi_{B}=\pi$, there must be at least two points where the nodal line passes through this 2D slice. Therefore, the inversion eigenvalues at all eight TRIMs indicate how many times (modulo four) a given 2D slice in the $3 \mathrm{D} \mathrm{BZ}$ is passed by nodal lines.

In this section, we also need to diagnose nodal lines in the presence of other symmetries in addition to inversion, where a simple extension of the lemma is incurred. In the presence of mirror symmetry (denoted by $M$ ), in addition to inversion symmetry, there are special 2D planes in the BZ where $M \mathbf{k}=\mathbf{k}$. On these mirror-invariant planes, thanks to $\hat{M}^{2}=+1$ and $[\hat{M}, \hat{P}]=[\hat{M}, \hat{T}]=0$, we can separate all bands into the $\hat{M}=+1$ sector and $\hat{M}=-1$ sector, and the bands in each sector have their respective inversion eigenvalues at the four TRIMs, as well as their respective Berry phases associate with the loop in Fig. 1(a).

Lemma: First variation.-

$$
e^{i \Phi_{B}^{( \pm)}}=\prod_{n \in \mathrm{occ}, K \in \mathrm{TRIM}} \chi_{n}^{ \pm}(K) .
$$

If both $\Phi_{B}^{(+)}$and $\Phi_{B}^{(-)}$are $\pi$, then there are two Dirac points in the +1 sector and two in the -1 sector. The Dirac points in opposite sectors may appear at the same momenta via fine-tuning but cannot pairwise annihilate each other, as their hybridization is disallowed by mirror symmetry.

We now apply the lemma and its variations (more to be introduced later) to study the band topology of each centrosymmetric space group that has a nontrivial indicator group. These 41 space groups [25] are further divided into six classes according to the presence of rotation and/or screw axes. Within each class, we first write down the explicit expressions of all indicators, followed by analyzing the band crossings for all combinations of nonzero indicators. The minimal possible configurations of nodal lines for each nonzero set of indicators for each of the 41 space groups are tabulated in Table III, where "minimal" means that we have tried to minimize the number of lines among all possible configurations.

\section{A. Space group 2}

The indicator group for space group 2 is $\mathbb{Z}_{2} \times \mathbb{Z}_{2} \times \mathbb{Z}_{2} \times \mathbb{Z}_{4}$, and the corresponding indicator set $\left(z_{2,1} z_{2,2} z_{2,3} z_{4}\right)$ is defined in Eq. (4). The definitions of $z_{2, i=1,2,3}$ are identical to the expressions for the weak indicators in the original Fu-Kane formula, only that, in the original context, their nonzero values imply weak topological insulators. The definition of $z_{4}$ may look both familiar and strange: $z_{4} \bmod 2$ is the familiar strong indicator in the original Fu-Kane formula, whose nonzero value implies a strong topological insulator if SOC is 
TABLE III. Minimal configurations of nodal lines in centrosymmetric space groups, given any nonzero set of symmetry-based indicators. The first column is the class of space groups by which the text is broken into subsections. The second column is the indicators we choose for the generators of the indicator group. The third column is for the numbers of space groups. The fourth column gives the Bravais lattice and the Brillouin zone. The reciprocal lattice $\left(\mathbf{g}_{i=1,2,3}\right)$ setting and convention for the Brillouin zone follow Ref. [31]. The fifth column contains all possible combinations of nonzero indicators and their corresponding configurations, where blue lines represent nodal rings without $\mathbb{Z}_{2}$-monopole charge, yellow lines represent nodal rings with $\mathbb{Z}_{2}$-monopole charge, gray planes are mirror planes at which nodal rings are centered, and red lines are the rotation or screw axes surrounded by one or two nodal rings. Note here that if two rings or lines are related by a reciprocal vector, only one of them is drawn.

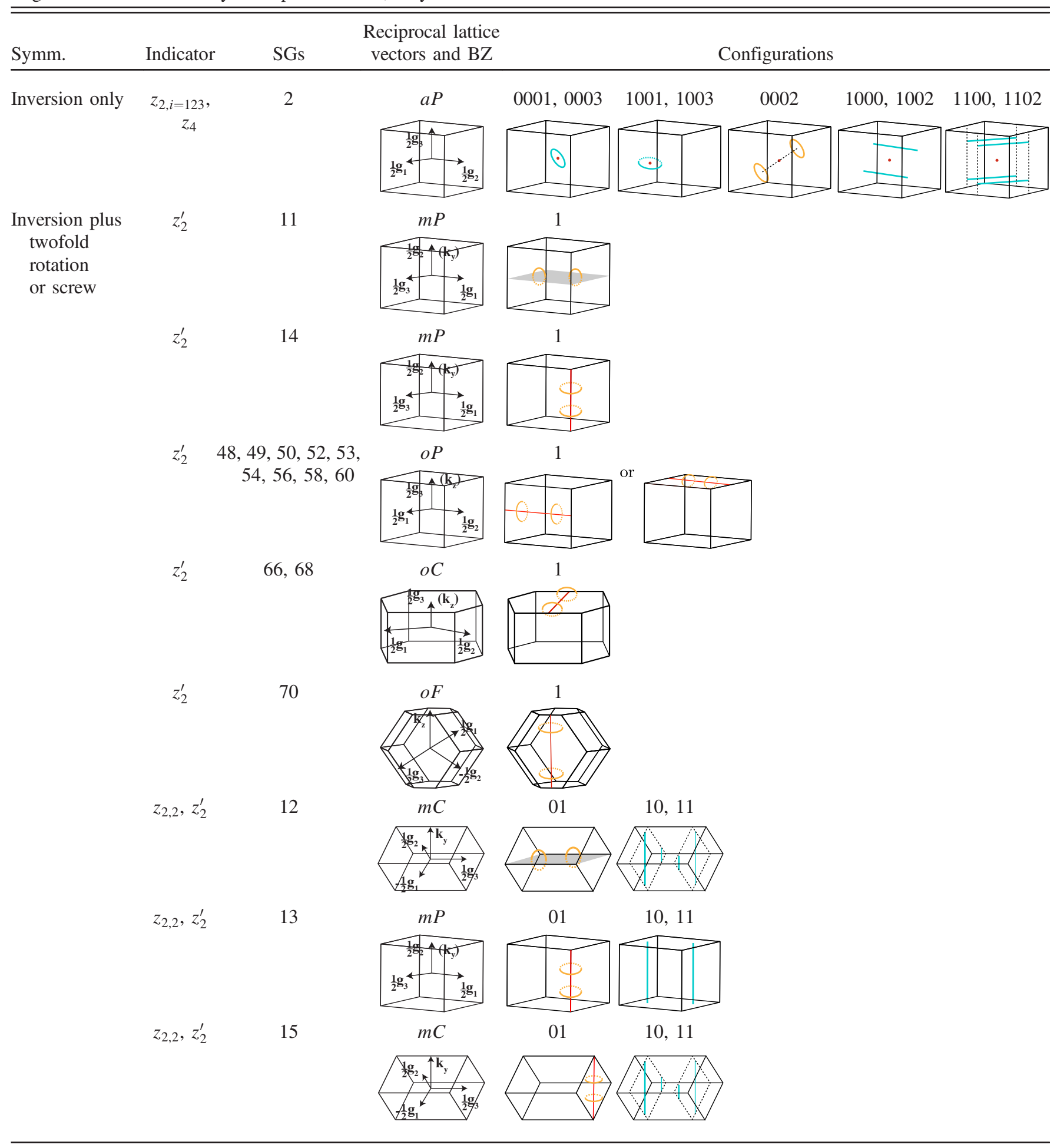


TABLE III. (Continued)

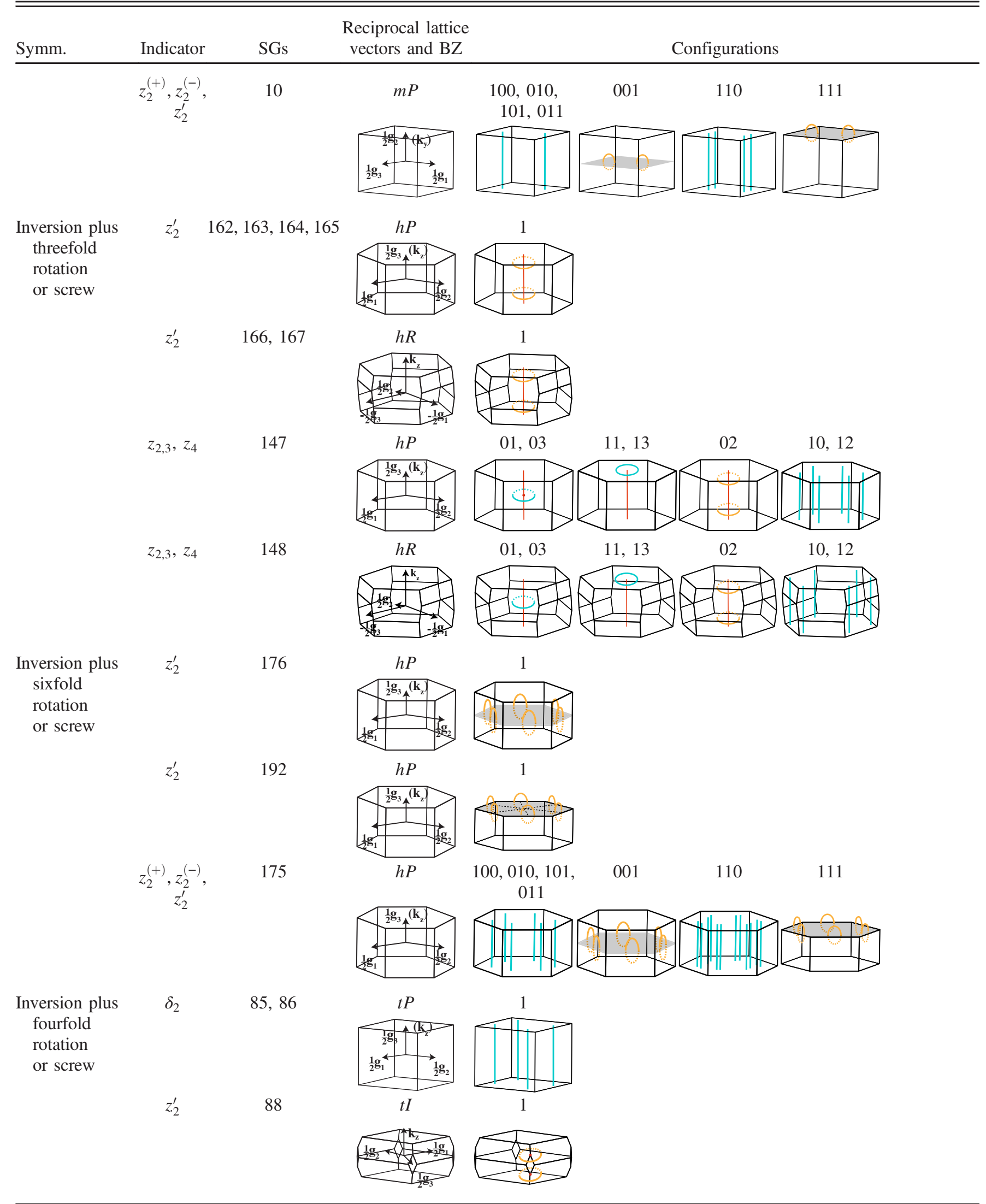


TABLE III. (Continued)

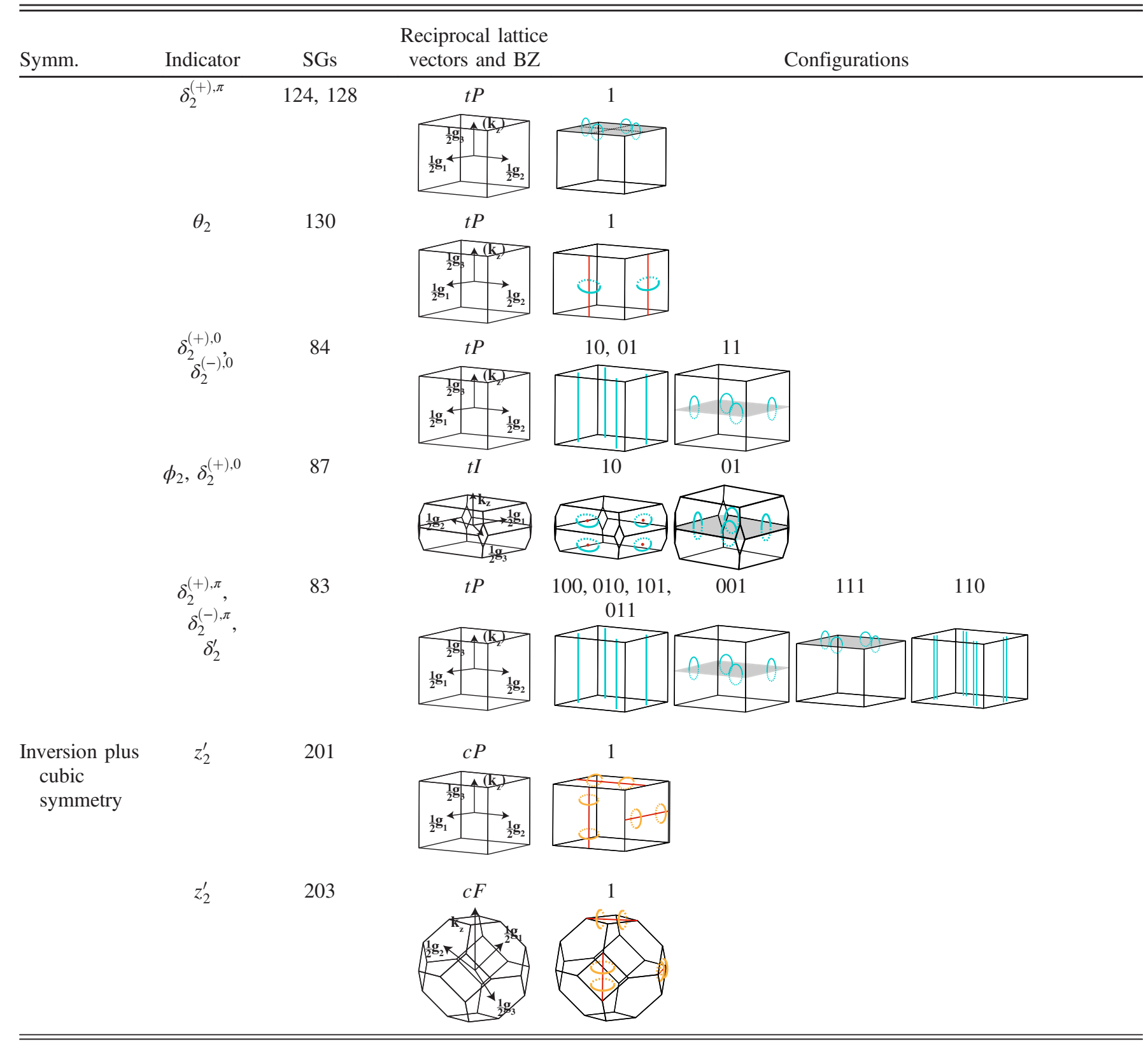

present, so it is unsurprising if $z_{4}=1,3$ gives us some topological semimetal without SOC, while the physical meaning of $z_{4}=2$ remains far from clear at this point.

Before analyzing the implications of nonzero $z_{2, i}$ and $z_{4}$, we remark that $z_{2, i}$ 's are "weak," in the sense that they change value under translation-breaking perturbations that preserve inversion symmetry, but that $z_{4}$ is a strong indicator. Translation-breaking perturbations, such as density-wave order parameters, effectively fold the BZ such that all eight TRIMs in the unfolded BZ are now at $\Gamma$ and that each new TRIM (which were interior points of the original BZ) has the same number of bands having positive parity as that of bands having negative parity. Therefore, after this folding, it is easy to confirm that $z_{2, i}=0$ in the folded BZ. It is also easy to check that $z_{4}$ remains the same after this folding. Intuitively, this result is because, in its expression, all TRIMs contribute equally.

Invoking the lemma in Eq. (8), we can establish the following statements regarding which planes are crossed by nodal lines for how many times. (i) If $z_{4}=0,2$ and $z_{2, i}=1$, both the slice at $k_{i}=0$ and $k_{i}=\pi$ are crossed by nodal lines for 2 mod 4 times, and the crossing points are pairwise related by time reversal, where $k_{i}$ is the component of the momentum when decomposed into three reciprocal lattice vectors, i.e., $\mathbf{k}=\sum_{i=1,2,3} k_{i} \mathbf{b}_{i}$. (ii) If $z_{4}=1,3$ and $z_{2, i}=0$, the slice at $k_{i}=0$ is crossed by nodal lines for 2 mod 4 times, so that a possible configuration of nodal lines is a single nodal ring that is symmetric about $\Gamma$. (iii) If 
$z_{4}=1,3$ and $z_{2, i}=1$, the slice at $k_{i}=\pi$ is crossed by nodal lines for 2 mod 4 times. In summary, if $z_{4}=0,2$ and some $z_{2, i} \neq 0$, a possible configuration of nodal lines has two lines, related by time reversal, that run along the direction of $\sum_{i} z_{2, i} \mathbf{b}_{i}$, and if $z_{4}=1,3$, a possible configuration is a single nodal ring around the TRIM at $\sum_{i} z_{2, i} \mathbf{b}_{i} / 2$.

The analysis above shows that $z_{4}=0, z_{2, i}=1$ and $z_{4}=2, z_{2, i}=1$ correspond to the same nodal-line configuration. Indeed, this result must be true, because the indicator sets $z_{4}=0, z_{2, i}=1$ and $z_{4}=2, z_{2, i}=1$ change to each other upon changing the inversion center used to define parity (Sec. II B), which leaves all physical observables invariant. However, it should be noted that, given a fixed inversion center, a state with $z_{4}=0, z_{2, i}=1$ and a state with $z_{4}=2, z_{2, i}=1$ have a relative difference, since by definition we cannot tune one to another without closing gaps at high-symmetry momenta. This relative difference can be detected by additional gapless modes (in addition to the bulk modes) on a domain wall of the two states, provided that the configuration keeps inversion symmetry.

There is one and only one remaining nonzero set of indicators, where $\left(z_{2,1} z_{2,2} z_{2,3} z_{4}\right)=(0002)$. Using the lemma, we find that there is no plane that must be crossed by nodal lines, so that the natural question becomes: Could this state be a (topological) gapped state? To answer this question, we first look at a related question: Is a double copy of the (0001) state a gapped state? According to the above results, the (0001) state has one nodal ring around $\Gamma$, crossing the $k_{3}=0$ plane at $\pm \mathbf{k}_{0}$. By doubling the state of (0001), we have also doubled the nodal rings. Then, we introduce coupling between the two copies while preserving $P$ and $T$. Illustrated in Fig. 2, the two points on the plane $k_{3}=0$ where the two rings cross the plane can be gapped, and now we have two disconnected loops on each side of the plane. We now show that each loop has nonzero $\mathbb{Z}_{2}$-monopole charge [35] and is therefore topologically stable against any inversion-preserving perturbations. The $\mathbb{Z}_{2}$-monopole charge is defined on a closed 2D manifold, e.g., a sphere, that encloses the nodal loop, originating from the nontrivial second homotopy group of real Grassmannian manifolds. There has not been any prediction
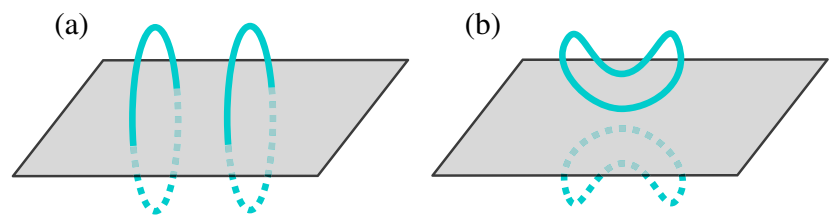

FIG. 2. (a) Two nodal rings that are contributed by the two copies of the (0001) state have two loops at the same position (deliberately separated for distinction), each crossing the $k_{3}=0$ plane. (b) Adding a hybridization between the two copies can open a full gap on the $k_{3}=0$ plane, which results in a reconnection of the nodal structure, such that, on each side of $k_{3}=0$, there is a nodal ring with nontrivial $\mathbb{Z}_{2}$-monopole charge. of electronic materials hosting this new type of nodal line, probably due to the involved calculation of its topological invariant. To show that the nodal loops in Fig. 2(b) carry nonzero $\mathbb{Z}_{2}$ charge, one should closely study how the crossing points on the $k_{3}=0$ plane are gapped out. Near $\mathbf{k}_{0}$, where the nodal loop crosses the $k_{3}=0$ plane, the effective theory of a $z_{4}=1$ state on the $k_{3}=0$ plane takes the Dirac form

$$
h\left(q_{1}, q_{2}\right)=q_{1} \sigma_{x}+q_{2} \sigma_{z},
$$

where $\mathbf{q} \equiv \mathbf{k}-\mathbf{k}_{0}, \sigma_{i}$ 's are Pauli matrices, and we implicitly choose the symmetry representation $\hat{P} \hat{T}=K$, where $K$ means complex conjugation. Doubling the whole system automatically doubles $h(\mathbf{q})$ to $H(\mathbf{q})=h(\mathbf{q}) \oplus h(\mathbf{q})$. For the four-band model $H(\mathbf{q})$, there is only one term that gaps the spectrum while preserving $P T: m \tau_{y} \sigma_{y}$, where $\tau_{z}= \pm 1$ is the flavor index. Because of time-reversal symmetry, the two Dirac points at $-\mathbf{k}_{0}$ also gap out each other such that the plane $k_{3}=0$ becomes fully gapped, where the $\mathbb{Z}_{2}$ invariant $\nu$ protected by $P T$ symmetry can be defined. For four-band models of the form

$$
H(\mathbf{k})=d_{x}(\mathbf{k}) \tau_{0} \sigma_{x}+d_{y}(\mathbf{k}) \tau_{0} \sigma_{z}+d_{z}(\mathbf{k}) \tau_{y} \sigma_{y},
$$

the $\mathbb{Z}_{2}$ invariant is given by [35]

$$
\nu=\frac{1}{4 \pi} \iint_{\mathrm{BZ}} d k^{2} \hat{d} \times \partial_{k_{1}} \hat{d} \times \partial_{k_{2}} \hat{d} \bmod 2,
$$

where $d_{i}(\mathbf{k})$ are real functions and $\hat{d}$ the unit vector of $\left(d_{x}, d_{y}, d_{z}\right)$. Inserting $H(\mathbf{q})$ into the integral in Eq. (12), one finds that $H(\mathbf{q})$ contributes $1 / 2$ to $\nu$. To obtain the integral near $-\mathbf{k}_{0}$, we first notice that the Bloch wave functions at $-\mathbf{k}_{0}$ can be obtained by acting $T$ on the wave functions at $\mathbf{k}_{0}$, so as to fix the basis vectors for the $k \cdot p$ theory near $-\mathbf{k}_{0}$. In this basis, the effective theory at $-\mathbf{k}_{0}$ is simply $H^{\prime}(\mathbf{q})=H(-\mathbf{q})$. An explicit calculation shows that the contribution of $H^{\prime}(\mathbf{q})$ is also $1 / 2$, and $\nu$ for entire the $k_{3}=0$ slice is $\nu\left(k_{z}=0\right)=1$. On the other hand, $\nu\left(k_{z}=\pi\right)$ for the $k_{3}=\pi$ slice is trivial: Before doubling, the $k_{3}=\pi$ plane is gapped and may possess either a trivial or nontrivial $\nu$, but, due to its $\mathbb{Z}_{2}$ nature, after doubling $\nu$ simply vanishes. Therefore, the difference of $\nu$ between the $k_{3}=0$ and $k_{3}=\pi$ planes implies that there is a nodal loop with nontrivial $\mathbb{Z}_{2}$ charge on each side of the $k_{3}=0$ plane.

While the doubled (0001) state is a (0002) state, the inverse is not necessarily true. Two states with the same indicators (0002) may, in principle, still be topologically distinct, and we cannot exclude the possibility that there are (0002) states that are fully gapped. Nevertheless, we argue that this is highly unlikely based on the following observation. Suppose we start from the (0002) state built from doubling a (0001) state. In order to gap out the two nodal loops, they have to pairwise annihilate each other at a TRIM, which we assume to be $\Gamma$ without the loss of 
generality. Consider a four-band $k \cdot p$ model near $\Gamma$ that describes this annihilation process:

$$
H=k_{1} \tau_{0} \sigma_{x}+k_{2} \tau_{0} \sigma_{z}+k_{3} \tau_{z} \sigma_{0}+\left(m-k_{z}^{2}\right) \tau_{y} \sigma_{y},
$$

where the symmetry operators are represented by $\hat{P}=\tau_{y} \sigma_{y}$ and $\hat{T}=K \tau_{y} \sigma_{y}$. The two loops with $\mathbb{Z}_{2}$ charge are, if $m>0$,

$L_{ \pm} \equiv\left\{\left(k_{1}, k_{2}, k_{3}\right) \mid k_{3}= \pm \sqrt{m}, \sqrt{k_{1}^{2}+k_{2}^{2}}=m\right\}$.

At $m=0$, both loops shrink to a point and vanish at $\Gamma$, and the model is fully gapped for $m<0$. Therefore, the model indeed describes a topological transition from $m>0$ to $m<0$, where two loops having $\mathbb{Z}_{2}$ charge annihilate at $m=0$. For $m>0(m<0)$, the two occupied bands at $\Gamma$ have negative (positive) parity such that, after the band inversion, the strong index $z_{4}$ changes by two. Therefore, within a four-band model, it is impossible for a (0002) state to be fully gapped. For a system of more than four bands, we can reasonably assume that, when the two nodal loops are sufficiently close to each other, the bands away from the Fermi energy can be energetically separated from the four bands involved in the annihilation without changing band topology, so that the above analysis remains valid.

To further confirm the correspondence between $z_{4}=2$ and nodal loops with $\mathbb{Z}_{2}$ charge, we generalize the $k \cdot p$ model in Eq. (13) to a tight-binding model given by

$$
\begin{aligned}
H(\mathbf{k})= & \sin k_{1} \tau_{0} \sigma_{x}+\sin k_{2} \tau_{0} \sigma_{z}+\sin k_{3} \tau_{z} \sigma_{0} \\
& +\left(\Delta-\sum_{i} \cos k_{i}\right) \tau_{y} \sigma_{y}
\end{aligned}
$$

and the symmetry operators are still $\hat{P}=\tau_{y} \sigma_{y}$ and $\hat{T}=K \tau_{y} \sigma_{y}$. On one hand, the $\mathbb{Z}_{2}$ invariant [Eq. (12)] of a $k_{z}$ plane can be explicitly derived as

$$
\nu\left(k_{z}\right)=\operatorname{sgn}\left[\left(\Delta-\cos k_{3}-2\right)\left(\Delta-\cos k_{3}+2\right)\right] \bmod 2 .
$$

The $\mathbb{Z}_{2}$ charge in half of the BZ, given by the difference of $\nu\left(k_{3}=0\right)$ and $\nu\left(k_{3}=\pi\right)$, can be expressed as $\operatorname{sgn}(\Delta-3)$ $(\Delta-1)(\Delta+1)(\Delta+3) \bmod 2$ [35]. Therefore, in half of the BZ exists one $(\bmod 2)$ nodal loop with $\mathbb{Z}_{2}$ charge when $1<|\Delta|<3$. On the other hand, it is direct to verify that for $1<|\Delta|<3$ the indicator set of the lower two bands is (0002), whereas for $|\Delta|>3$ or $|\Delta|<1$ the indicator set is (0000). Therefore, the correspondence between $z_{4}=2$ and nodal loops with $\mathbb{Z}_{2}$ charge still holds in this tightbinding model.

Up to this point, we have used both mathematics and physical arguments to support the conclusion that a state with indicators (0002) must have one (or an odd number of) nodal ring(s) in each half of the BZ, and each nodal ring has nonzero $\mathbb{Z}_{2}$-monopole charge. This concludes the analysis of each nonzero set of indicators for space group 2. Before going to the next part, we briefly comment on the difficulty in finding a rigorous proof for the relation between indicator set (0002) and the presence of a $Z_{2}$-charged nodal ring. One may attempt at relating the $Z_{2}$ invariant of a 2D plane with $P$ and $T$ to the inversion eigenvalues and then show that, if $k_{z}=0$ and $k_{z}=\pi$ have different $Z_{2}$, there must be one nodal ring having $Z_{2}$-monopole charge. The same proof works for the relation between the number of Weyl points and the inversion eigenvalues in 3D with inversion but not time reversal. However, in our case, one may easily prove that $2 \mathrm{D}$ systems having $P$ and $T$ do $n o t$ have any indicator, so that there does not exist any relation between the $Z_{2}$ invariant and the inversion eigenvalues.

\section{B. Inversion plus twofold rotation or screw axis}

From this point, we start adding one additional rotation or screw axis to enhance the space group 2 to a higher space group. As convention, the direction of the highest-order rotation (screw) axis is defined as the $z$ direction.

There are 18 space groups that have (i) at least one twofold rotation or screw axis, (ii) no higher-order rotation or screw axis, and (iii) a nontrivial indicator group. Two observations relate their indicators to the indicators of group 2: (i) Among the 18, space group 14 is their common subgroup, and a study of the compatibility relation in group 14 reveals that $z_{4} \in$ even; (ii) all 18 space groups are subgroups of space group 192, and the compatibility relations of group 192 allows at least one solution having $z_{4}=2$. These two facts together give at least one strong $\mathbb{Z}_{2}$ indicator shared by all 18 space groups: $z_{2}^{\prime} \equiv z_{4} / 2$.

The subsection, hence, is further divided into parts by the number of independent $\mathbb{Z}_{2}$ indicators other than $z_{2}^{\prime}$.

\section{1. $\mathbb{Z}_{2}$ indicators in space groups $11,14,48,49,50$, $52,53,54,56,58,60,66,68$, and 70}

Since $z_{2}^{\prime}$ is always an indicator and the 14 space groups in this part have only one indicator, this indicator must be $z_{2}^{\prime}$. As group 2 is their common subgroup, when $z_{2}^{\prime}=1$, there is one (or an odd number of) nodal ring in each half of the BZ, but their configurations now are further constrained by rotation or screw axes.

Finding the constraints requires a detailed study of the $k \cdot p$ model of a nodal ring having $\mathbb{Z}_{2}$-monopole charge. The $k \cdot p$ model expanded about the center of a nodal ring with $\mathbb{Z}_{2}$-monopole charge is [35]

$$
h(\mathbf{q})=q_{1} \sigma_{x}+q_{2} \sigma_{z}+q_{3} \tau_{y} \sigma_{y}+m \tau_{z} \sigma_{0},
$$

where the only assumed symmetry $P T$ is represented by $\hat{P} \hat{T}=K$. The Hamiltonian in Eq. (17) gives a nodal ring of radius $\sqrt{|m|}$ perpendicular to the $q_{3}$ direction. It is easy to 
confirm that $h(\mathbf{q})$ is consistent with an additional twofold rotation $\hat{C}_{2}=\sigma_{y}$ satisfying $C_{2}^{2}=1$ such that

$$
\hat{C}_{2}^{-1} h\left(q_{1}, q_{2}, q_{3}\right) \hat{C}_{2}=h\left(-q_{1},-q_{2}, q_{3}\right) .
$$

It is important to notice that $\hat{C}_{2}$ thus defined anticommutes with $\hat{P} \hat{T}$ and that it is impossible to find any Dirac matrix that commutes with $P T$ while satisfying Eq. (18). Therefore, the nodal ring may be centered at a $C_{2}$ axis where

$$
\hat{C}_{2} \hat{P} \hat{T}=-\hat{P} \hat{T} \hat{C}_{2}
$$

This equation is satisfied only if, (i) in real space, the $C_{2}$ axis does not pass any inversion center, and (ii) at the $C_{2^{-}}$ invariant momentum, commuting $P$ and $C_{2}$ generates a minus sign. In any lattice having twofold rotation and inversion, we can set the inversion center at (000) and the rotation axis as the line passing through $\mathbf{t} / 2$, where $\mathbf{t}$ is a vector perpendicular to the rotation axis such that $C_{2}=$ $\{2 \mid \mathbf{t}\}$ and $C_{2} P=\{1 \mid 2 \mathbf{t}\} P C_{2}$. Then, for a $C_{2}$-invariant line in momentum space, if it satisfies $2 \mathbf{k} \cdot \mathbf{t}=\pi \bmod 2 \pi$, Eq. (19) holds. Similar results apply for twofold screw axis $C_{2_{1}}=\{2 \mid \mathbf{t}+\mathbf{s}\}$, where $\mathbf{s}$ is the screw-vector parallel with screw axis, but the anticommutation is replaced by

$$
\hat{C}_{2_{1}} \hat{P} \hat{T}=-e^{2 i s \cdot \mathbf{k}} \hat{P} \hat{T} \hat{C}_{2_{1}}
$$

Applying these results to the listed space groups, we find that space groups $14,48,49,50,52,53,54,56,58,60,66$, 68 , and 70 have twofold axes not passing through any inversion center, and we plot in Table III nodal rings around one $C_{2^{-}}$or $C_{2_{1}}$-invariant line where either Eq. (19) or Eq. (20) is met. In space group 11, where the screw axis contains an inversion center, each of the two nodal rings is centered and symmetric about the $k_{2}=0$ plane, and the two rings are related to each other under the twofold screw rotation. (For group 11, the screw axis is along the $k_{2}$ direction, as shown in Table III.)

\section{2. $\mathbb{Z}_{2} \times \mathbb{Z}_{2}$ indicators in space groups 12,13 , and 15}

Other than the $\mathbb{Z}_{2}$ indicator $z_{2}^{\prime}$, there is another $\mathbb{Z}_{2}$ indicator for these three monoclinic space groups. An analysis of the compatibility relations reveals that this is nothing but the second weak index $z_{2,2}$. When $z_{2,2}=1$, the strong indicator $z_{2}^{\prime}$ becomes convention dependent (see discussion in Secs. II B and III A), and the nodal lines are two lines running along the $k_{2}$ direction, related to each other under time reversal. When $z_{2,2}=0$ and $z_{2}^{\prime}=1$, there are again two nodal rings having $\mathbb{Z}_{2}$-monopole charge, the positions of which depend on whether the rotation axis contains any inversion center, as explained in Sec. III B 1.

\section{3. $\mathbb{Z}_{2} \times \mathbb{Z}_{2} \times \mathbb{Z}_{2}$ indicators in space group 10}

Space group 10 has a simple monoclinic lattice with a twofold rotation axis, so that $P$ and $C_{2}$ imply a mirror plane $M \equiv P C_{2}$ that also contains the inversion center. In this case, the $2 \mathrm{D}$ slice at $k_{2}=\pi$ is a mirror invariant plane in the BZ, on which we can define two $\mathbb{Z}_{2}$ indicators for the $M=+1$ and $M=-1$ sectors as $z_{2}^{(+)}$and $z_{2}^{(-)}$[Eq. (7)], respectively. From Eqs. (4) and (7), we see that the previously defined index $z_{2,2}$ satisfies

$$
z_{2,2}=z_{2}^{(+)}+z_{2}^{(-)} \bmod 2 .
$$

Therefore, if $z_{2}^{(+)}=1$ and $z_{2}^{(-)}=0$ (or vice versa), there are two nodal lines running along the $k_{2}$ direction, related to each other by time reversal. We are now left with three cases: (a) $z_{2}^{( \pm)}=0, z_{2}^{\prime}=1$, (b) $z_{2}^{( \pm)}=1, z_{2}^{\prime}=0$, and (c) $z_{2}^{( \pm)}=1, z_{2}^{\prime}=1$

(a) $z_{2}^{( \pm)}=0$ means that $k_{2}=\pi$ plane is crossed by $0 \mathrm{mod}$ 4 nodal lines, and $z_{2}^{\prime}=1$ means that there are in the entire BZ 2 mod 4 nodal rings. According to the discussion in Sec. III B 1, since the rotation axis contains at least one inversion center, a nodal ring cannot be symmetric about any $C_{2}$-invariant line. The only possibility is that both nodal rings are vertical, symmetric about the $k_{2}=0$ plane, but related to each other under the twofold rotation.

(b) $z_{2}^{( \pm)}=1$ means that the nodal lines cross the $k_{z}=\pi$ plane four times, two in the $M=+1$ and two in the $M=-1$ sectors, respectively, while $z_{2}^{\prime}=0$ means that the same happens on the $k_{0}=0$ plane. A typical configuration has in total four nodal lines running along the $k_{2}$ direction.

(c) $z_{2}^{( \pm)}=1$ means that the nodal lines cross the $k_{z}=\pi$ plane four times, two in the $M=+1$ and two in the $M=-1$ sectors, respectively, while $z_{2}^{\prime}=1$ means there are in total 2 mod 4 nodal rings in the entire BZ. A typical configuration has two vertical nodal rings, related to each other by twofold rotation, but now centered and symmetric about the $k_{2}=\pi$ plane.

\section{Inversion plus threefold rotation or screw axis}

Now we consider, instead of a twofold axis, adding a threefold or screw rotation axis to space group 2. Among all space groups having these two symmetries, the following eight have nontrivial indicator group: 147, 148, and 162-167. All eight are either subgroups of group 166 or 192 , both having at least one band structure that gives $z_{4}=2$. Therefore, they at least have a strong $\mathbb{Z}_{2}$ indicator $z_{2}^{\prime}=z_{4} / 2$. In fact, it is the only indicator for space groups $162-167$, where the indicator group is $\mathbb{Z}_{2}$. When $z_{2}^{\prime}=1$, there are $2 \bmod 4$ nodal rings, and there their configuration must observe both $C_{3}$ and time-reversal symmetries. Unlike twofold axes, threefold axes always contain inversion 
centers in these space groups. A study of the model in Eq. (17) shows that we can define

$$
\hat{C}_{3}=-\exp \left(i \sigma_{y} \pi / 3\right),
$$

which satisfies $\hat{C}_{3}^{3}=1,\left[\hat{C}_{3}, \hat{P} \hat{T}\right]=0$, and

$$
\hat{C}_{3}^{-1} h(\mathbf{k}) \hat{C}_{3}=h\left(C_{3} \mathbf{k}\right) .
$$

Therefore, while a nodal ring having a $\mathbb{Z}_{2}$-monopole charge is inconsistent with a twofold rotation through its center if $\left[\hat{C}_{2}, \hat{P} \hat{T}\right]=0$, it is consistent with a threefold rotation, so that a ring may be surrounding a $C_{3}$-invariant line in the BZ.

\section{1. $\mathbb{Z}_{2}$ indicators in space groups 162-167}

As said, $z_{2}^{\prime}$ is the only indicator for these groups, and if $z_{2}^{\prime}=1$, there are two nodal rings, each of which is symmetric about a $C_{3}$-invariant line in the $\mathrm{BZ}$, that are related to each other by time reversal.

\section{2. $\mathbb{Z}_{2} \times \mathbb{Z}_{4}$ indicators in space groups 147 and 148}

These two space groups have higher-symmetry indicators. In fact, they allow $z_{4}$ defined in Eq. (4) to take all four possible values and also allow the three weak indicators to be the same and nonzero, i.e., $z_{2,1}=z_{2,2}=z_{2,3}=1$. The topological information indicated by their nonzero combinations is identical to those discussed in Sec. III A, with the only difference that, due to the threefold axis, the state having $z_{2,3}=1$ and $z_{4}=0,2$ should have $6 \bmod 12$ nodal lines running in the third direction.

\section{Inversion plus six rotation or screw axes}

There are only three space groups that have a sixfold axis and nontrivial indicator group, all of which are subgroups of space group 192. Group 192 allows at least one band structure having $z_{4}=2$ such that the three groups have at least one strong $\mathbb{Z}_{2}$ indicator, $z_{2}^{\prime} \equiv z_{4} / 2$. When $z_{2}^{\prime}=1$, there must be $2 \bmod 4$ nodal rings protected by $P$ and $T$, and the additional symmetries put further constraints on their numbers and configurations. Like threefold axes, sixfold axes always contain inversion centers, and, since a sixfold axis is automatically a twofold axis, all twofold axes also contain inversion centers in the three subgroups. According to the discussion in Sec. III B 1, any nodal ring in this case cannot be symmetric about a $C_{2}$-invariant line or a $C_{6}$-invariant line. A minimal configuration, having the least number of rings, therefore has six nodal rings, each vertical and symmetric under the mirror symmetry $k_{z} \rightarrow-k_{z}$. They are related to each other by a sixfold rotation or screw and may be centered on either the $k_{z}=0$ or the $k_{z}=\pi$ plane (depending on which space group). In all three space groups, the mirror plane allows us to define the two additional $\mathbb{Z}_{2}$ indicators, as in the twofold axis case, $z_{2}^{( \pm)}$for the positive and negative mirror sectors, respectively.

\section{1. $\mathbb{Z}_{2}$ indicators in space groups 176 and 192}

For groups 176 and 192, $z_{2}^{\prime}$ is the only indicator, and its nontrivial value corresponds to the six-ring configuration described above. However, in group 176, the screw axis makes the entire $k_{z}=\pi$ plane double degenerate, inconsistent with having 12 discrete band touch points where the six rings cross this plane. These rings are hence centered at the $k_{z}=0$ plane, each symmetric about the plane. In group 192, the nontrivial indicator always corresponds to band structure having $z_{2}^{( \pm)}=1$, which means that the six rings are located on the $k_{z}=\pi$ plane.

\section{2. $\mathbb{Z}_{2} \times \mathbb{Z}_{2} \times \mathbb{Z}_{2}$ in space group 175}

The three indicators forming the indicator group of space group 175 are $z_{2}^{( \pm)}$and $z_{2}^{\prime}$. The topological information carried by their nonzero combinations can be derived in the same way as in Sec. III B 3.

\section{E. Inversion plus additional fourfold rotation or screw axis}

We now consider adding fourfold rotation or screw axes to space group 2 and also to space groups having twofold axes: These are the tetragonal space groups.

Among them, the following ones have nontrivial indicator groups: $83-88,124,128$, and 130, nine in total. A calculation shows that only groups 86 and 88 allow band structures having $z_{4}=2$, while all the others have $z_{4}=0$. Therefore, for the other seven space groups, three new $\mathbb{Z}_{2}$ indicators are found.

Before defining them, we need to introduce a new variation of the lemma presented in Eq. (9). Consider a 2D system having $C_{4}, P$, and $T$, and consider a loop shown in Fig. 1(b), which encloses a quadrant of the BZ. It is shown in Ref. [36] that the Berry phase associated with this loop is determined by the symmetry eigenvalues of the occupied bands in the following equation.

Lemma: Second variation.-

$$
\exp \left(i \Phi_{B}\right)=\prod_{n \in \text { occ }} \xi_{n}(\Gamma) \xi_{n}(M) \zeta_{n}(X),
$$

where $\xi$ is the $C_{4}$-rotation eigenvalue and $\zeta$ the $C_{2}$-rotation eigenvalue. While $\xi_{n}$ takes four possible values \pm 1 and $\pm i$, time reversal ensures that $\pm i$ appear in pairs, such that the rhs of Eq. (24) can be only either +1 or -1 . If -1 , we have $\Phi_{B}=\pi$, and, since $P T$ makes Berry curvature vanish, this result implies that in each quadrant of the BZ there must be $1 \bmod 2$ Dirac points.

We can apply this lemma to 3D systems having the same symmetries. For the $k_{z}=0$ and the $k_{z}=\pi$ slice in a simple tetragonal lattice and for the $k_{z}=0$ slice in a body-centered 
tetragonal lattice, applying the lemma of Eq. (24) simply tells us that, if $\Psi_{B}=\pi$, the slice of question is crossed by nodal lines 1 mod 2 times in each quadrant or $4 \bmod 8$ times in total. This observation enables the definition of a new $\mathbb{Z}_{2}$ indicator:

$\delta_{2} \equiv N_{\xi=-1}(\Gamma)+N_{\xi=-1}(M)+N_{\zeta=-1}(X) \bmod 2$,

where $N_{\xi=-1}$ and $N_{\zeta=-1}$ are the numbers of occupied bands having $\xi=-1$ and $\zeta=-1$, respectively.

If the $C_{4}$ axis contains inversion centers, then $M=C_{4}^{2} P$ is a mirror plane. In this case, the Hamiltonian on the $k_{z}=0$ and $k_{z}=\pi$ slices can be further divided into decoupled sectors having $M=+1$ and $M=-1$, so that the Berry phase for the two sectors can also be separately defined on the two planes. Applying the lemma of Eq. (24) to each sector, we have the definitions of the following $\mathbb{Z}_{2}$ numbers:

$\delta_{2}^{( \pm), 0} \equiv N_{\xi=-1}^{( \pm)}(\Gamma)+N_{\xi=-1}^{( \pm)}(M)+N_{\zeta=-1}^{( \pm)}(X) \bmod 2$,

$\delta_{2}^{( \pm), \pi} \equiv N_{\xi=-1}^{( \pm)}(Z)+N_{\xi=-1}^{( \pm)}(A)+N_{\xi=-1}^{( \pm)}(R) \bmod 2$.

We emphasize that (i) if the lattice is body-centered tetragonal, then $\delta_{2}^{( \pm), \pi}$ is undefined, (ii) if $C_{4}$ is replaced by screw axis $C_{4_{1}}$, then again $\delta_{2}^{( \pm), \pi}$ is undefined, and (iii) these four numbers satisfy

$$
\delta_{2}^{(+), 0}+\delta_{2}^{(-), 0}=\delta_{2}^{(+), \pi}+\delta_{2}^{(-), \pi}=\delta_{2} \bmod 2 .
$$

Depending on the specific groups, we choose from these four indicators one, two, or three as generators of the indicator group.

\section{1. $\mathbb{Z}_{2}$ indicators in space groups $85,86,88$, 124,128 , and 130}

Unlike the cases of twofold or sixfold axes, here we cannot find a common $\mathbb{Z}_{2}$ indicator for these space groups, although their indicator groups are the same.

We directly solve the compatibility relations for all the basis vectors of the symmetry data space and check if $z_{2}^{\prime}$ or any of the above new indicators, namely, $\delta_{2}, \delta_{2}^{( \pm), 0 / \pi}$, is well defined in the given space group and, if yes, trivial or nontrivial. If any one of them is nontrivial for any one of the basis vectors of the band representation space, we know that this is the generator of the indicator group, because there is only one $\mathbb{Z}_{2}$ indicator.

The exhaustive search described above yields the indicator $\delta_{2}$ for groups 85 and 86 and the indicator $\delta_{2}^{(+), \pi}$ for groups 124 and 128. For group 130, the candidate indicators are either undefined or take a zero value for all possible basis vectors, and we have to solve some algebraic equations to find the expression of its indicator

$$
\begin{aligned}
\theta_{2}= & {\left[N\left(\Gamma_{1}^{+}\right)+N\left(\Gamma_{1}^{-}\right)+N\left(\Gamma_{3}^{+}\right)+N\left(\Gamma_{3}^{-}\right)\right.} \\
& \left.+N\left(M_{1}\right)+N\left(M_{2}\right)\right] / 2 \bmod 2,
\end{aligned}
$$

where $N$ (irrep) stands for the number of appearances of a certain irreducible representation in the valence bands at a given high-symmetry point. The labels of the irreducible representations follow the convention of Refs. [26,28,29].

The configurations of nodal lines are easily found for groups 85 and 86 . Since $\delta_{2}=1$, the nodal lines pass the $k_{z}=0$ plane for $4 \bmod 8$ times, but, since there is no band crossing along any high-symmetry line, the $C_{4}$ eigenvalues remain the same for any $k_{z}$ slice such that all planes with fixed $k_{z}$ are crossed by nodal lines at four points. Therefore, there must be four nodal lines running along the $k_{z}$ direction.

For groups 124 and 128, we find that if $\delta_{2}^{( \pm), \pi}=1$, then $\delta_{2}^{( \pm), 0}=0$ is guaranteed by the compatibility relations. Therefore, the nontrivial configuration has four nodal rings, each centered at and symmetric about the $k_{z}=\pi$ plane, related to each other by $C_{4}$.

For group 130, we notice that (i) it is a subgroup of group 124 and (ii) the band structure which corresponds to the nontrivial indicator $\delta_{2}^{(+), \pi}=1$ in group 124 gives $\theta_{2}=1$ when we reduce the symmetries of group 124 to those of group 130. These two facts mean that the configuration of nodal lines in group 124 is also a possible configuration in group 130 having $\theta_{2}=1$. The difference between the two space groups is that, while the four nodal rings must be centered on the $k_{z}=\pi$ plane in group 124, they can move to the positions shown in Table III: Two of the four are centered on $X R$ and two others on its $C_{4}$ equivalent line. Through this configuration, the four rings may further move to the $k_{z}=0$ plane. Based on this observation, we make a conjecture that $e^{i \theta_{2}}$ is, in fact, the Berry phase of the loop illustrated in Fig. 1(c).

The indicator for group 88 is, interestingly, the old inversion indicator $z_{2}^{\prime}$. If $z_{2}^{\prime}=1$, there should be $2 \bmod 4$ nodal rings. Point $P$ in the $\mathrm{BZ}$ is the only point whose multiplicity is two; therefore, in the minimal configuration, there are two rings around the two $P$ 's.

\section{2. $\mathbb{Z}_{2} \times \mathbb{Z}_{2}$ indicators in space groups 84 and 87}

An exhaustive search for indicators in space group 84 reveal the two $\mathbb{Z}_{2}$ indicators to be $\delta_{2}^{( \pm), 0}$. If $\delta_{2}^{(+), 0}=1$ and $\delta_{2}^{(-), 0}=0$, or if $\delta_{2}^{(-), 0}=1$ and $\delta_{2}^{(+), 0}=0$, there are four lines running along the $k_{z}$ direction. If $\delta_{2}^{(+), 0}=\delta_{2}^{(-), 0}=1$, there are four nodal rings centered and symmetric about the $k_{z}=0$ plane, and they are related to each other by the fourfold rotation or screw axis. 
For space group 87, one generator of the indicator group is found to be $\delta_{2}^{(+), 0}$, and, when $\delta_{2}^{(+), 0}=1$, the compatibility relations require also $\delta_{2}^{(-), 0}=1$. Since the rotation axis contains inversion centers, in the minimal configuration, there are four nodal rings centered at and symmetric about the $k_{z}=0$ plane, related to each other by the fourfold rotation. The other generator takes the form

$\phi_{2} \equiv N_{\xi=-1}(M)+N_{\chi=-1}(N)+N_{\xi^{\prime}=-1}(P) \bmod 2$,

where $N_{\xi=-1}(M)$ is the number of occupied states having $C_{4}$ eigenvalue -1 at $M, N_{\chi=-1}(N)$ is the number of occupied states having $P$ eigenvalue -1 at $N$, and $N_{\xi^{\prime}=-1}(P)$ is the number of states having $S_{4}$ eigenvalue -1 at $P$. We find that when $\phi_{2}=1$ the Berry phase along the loop shown in Fig. 3 is $\pi(\bmod 2 \pi)$, and, thus, $\phi_{2}=1$ corresponds to a semimetal where an odd number of nodal lines pass through the curve enclosed by the loop.

Now let us prove the correspondence between the Berry phase and the symmetry eigenvalues. Below, we adopt the notation

$$
W_{\mathbf{K} \rightarrow \mathbf{P}} \equiv \lim _{N \rightarrow \infty} U_{\mathbf{K}}^{\dagger} U_{\mathbf{k}_{1}} U_{\mathbf{k}_{1}}^{\dagger} U_{\mathbf{k}_{2}} \ldots U_{\mathbf{k}_{N}}^{\dagger} U_{\mathbf{P}}
$$

where $\mathbf{k}_{i=1 \ldots N}$ give a path from $\mathbf{K}$ to $\mathbf{P}, U_{\mathbf{k}}=\left[\left|u_{1, \mathbf{k}}\right\rangle\right.$, $\left.\left|u_{2, \mathbf{k}}\right\rangle \ldots\right]$, where each column represents an occupied state. Then, since wave functions are all real due to the $P T$ symmetry, the Berry phase along the loop in Fig. $3, \Phi_{B}$, is quantized to $0, \pi(\bmod 2 \pi)$ and given by

$e^{i \Phi_{B}}=\operatorname{det} W_{K_{3} \rightarrow M} W_{M \rightarrow K_{1}} W_{K_{1} \rightarrow P} W_{P \rightarrow K_{2}} W_{K_{2} \rightarrow K_{3}}$.

Without the loss of generality, we choose the gauge where wave functions in the path $K_{1} \rightarrow M$ (except for $C_{4^{-}}$ invariant point $M$ ) are $C_{4}$ rotation counterparts of the wave functions in the path $M \rightarrow K_{1}$, such as $U_{K_{1}}=C_{4} U_{K_{3}}$. For the $M$ point, we have $C_{4} U_{M}=U_{M} D^{M}\left(C_{4}\right)$, with $D^{M}\left(C_{4}\right)$ the $C_{4}$ representation matrix. Applying the $C_{4}$ operation on the wave functions in the path $W_{K_{3} \rightarrow M}$, we get

$W_{K_{3} \rightarrow M}=U_{K_{3}}^{\dagger} C_{4}^{\dagger} C_{4} \ldots C_{4}^{\dagger} C_{4} U_{M}=W_{M \rightarrow K_{1}}^{\dagger} D^{M}\left(C_{4}\right)$.

Taking similar gauges for other paths, we get

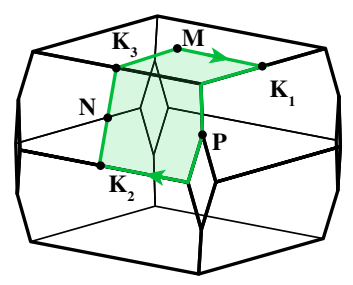

FIG. 3. A loop considered in a body-centered tetragonal lattice for space group 87 .

$$
W_{K_{1} \rightarrow P}=W_{P \rightarrow K_{2}}^{\dagger} D^{P}\left(S_{4}\right)
$$

and

$$
W_{K_{2} \rightarrow N}=W_{N \rightarrow K_{3}}^{\dagger} D^{N}(P),
$$

where $D^{P}\left(S_{4}\right)$ is the $S_{4}$ representation matrix at $P$ and $D^{N}(P)$ the $P$ representation matrix at $N$. Therefore, we have

$$
e^{i \Phi_{B}}=\operatorname{det} D^{M}\left(C_{4}\right) D^{P}\left(S_{4}\right) D^{N}(P) .
$$

Comparing Eqs. (30) and (36), we find that $\left(\Phi_{B} / \pi\right)=$ $\phi_{2} \bmod 2$.

\section{3. $\mathbb{Z}_{2} \times \mathbb{Z}_{2} \times \mathbb{Z}_{2}$ indicators in space group 83}

The three indicators for space group 83 are chosen to be $\delta_{2}^{( \pm), \pi}$ and $\delta_{2}^{\prime} \equiv \delta_{2}^{(+), 0}-\delta_{2}^{(+), \pi}=\delta_{2}^{(-), 0}-\delta_{2}^{(-), \pi}$, where the second equality is guaranteed by compatibility relations. The configurations corresponding to each nontrivial set of indicators are the following. If $\delta_{2}^{(+), \pi}=1$ and $\delta_{2}^{(-), \pi}=0$ or if $\delta_{2}^{(+), \pi}=0$ and $\delta_{2}^{(-), \pi}=1$, there are four nodal lines along the $k_{z}$ direction, related to each other by the fourfold rotation. In this case, the value of $\delta_{2}^{\prime}$ is irrelevant, as one can redefine the origin as $\mathbf{a}_{3} / 2$ such that the mirror eigenvalues at $k_{z}=\pi$ flip, leading to the interchange of $\delta^{(+) \pi}$ and $\delta^{(-) \pi}$ and, thus, the change of $\delta_{2}^{\prime}$. We can say the value of $\delta_{2}^{\prime}$ is "convention dependent" in the same way that $z_{4}=0$ and $z_{4}=2$ are convention dependent if any one of $z_{2, i} \neq 0$ (Secs. II B and III A). Similar to $z_{4}=0$ and $z_{4}=2$ in space group 2, in a fixed convention, $\delta_{2}^{\prime}=0$ and $\delta_{2}^{\prime}=1$ have a relative difference in the sense that we can tune one to another without closing gaps at high-symmetry momenta.

We are left with three cases: (i) $\delta_{2}^{(+), \pi}=\delta_{2}^{(-), \pi}=0$, $\delta_{2}^{\prime}=1$, where there are four nodal rings centered at and symmetric about the $k_{z}=0$ plane, related to each other under $C_{4}$; (ii) $\delta_{2}^{(+), \pi}=\delta_{2}^{(-), \pi}=1, \delta_{2}^{\prime}=0$, where there are eight nodal lines along the $k_{z}$ direction; and (iii) $\delta_{2}^{(+), \pi}=$ $\delta_{2}^{(-), \pi}=1, \quad \delta_{2}^{\prime}=1$, where there are four nodal rings centered at and symmetric about the $k_{z}=\pi$ plane, related to each other under $C_{4}$.

\section{F. Cubic space groups 201 and 203}

There are two space groups in the cubic system that have nontrivial indicator groups $\left(\mathbb{Z}_{2}\right)$. The indicator is simply $z_{2}^{\prime}=z_{4} / 2$, and, when $z_{2}^{\prime}=1$, there are $2 \bmod 4$ nodal rings. However, due to the presence of a threefold rotation axis, there would be in total 6 mod 12 nodal rings. In these space groups, none of the twofold axes contains any inversion center, so that each nodal ring is symmetric about a $C_{2}$ invariant line on the boundary of BZ where $\left\{\hat{C}_{2}, \hat{P} \hat{T}\right\}=0$. 


\section{NONCENTROSYMMETRIC SPACE GROUPS}

In the absence of inversion, the nodal lines are unprotected, so that all band crossings at generic momenta are Weyl points. There are only 12 noncentrosymmetric space groups that have nontrivial indicator groups. Except for group 81 , the indicator group is $\mathbb{Z}_{2}$, while for group 81 , the indicator group is $\mathbb{Z}_{2} \times \mathbb{Z}_{2}$. In this section, we use rotation eigenvalues at high-symmetry points to express these indicators, and for each nonzero indicator, we give one possible configuration of the Weyl points in the Brillouin zone. We have tried to provide the configuration with the minimal number of Weyl points.

This section is further divided into subsections, treating three classes of space groups according to the generators: a single rotation axis, or a rotation axis plus vertical glide planes, or an $S_{4}$ symmetry defined by $S_{4}:(x, y, z) \rightarrow(-y, x,-z)$, respectively. In Fig. 5, we provide the minimal configurations of Weyl points for each nonzero set of indicators in each one of the 12 space groups.

\section{A. Single rotation or screw axis (groups 3, 75, 77, 168, 171, and 172)}

These space groups have only one rotation $(3,75$, and $168)$ or screw $(77,171$, and 172$)$ axis. We can simply apply the lemma of Eq. (8) to the rotation (or screw) invariant planes in groups $3,168,171$, and 172 , where the product of all $C_{2}$ eigenvalues at all TRIMs implies whether there are 2 mod 4 band crossing points (Weyl points) in the plane. To be specific, we define for space groups $3,168,171$, and 172 the $\mathbb{Z}_{2}$ indicator

$$
\alpha_{2} \equiv \sum_{K_{i}=0, \mathbf{K} \in \mathrm{TRIM}} N_{\zeta=-1}(\mathbf{K}) \bmod 2,
$$

where $i=y$ for group 3 and $i=z$ for groups 168, 171, and 172. If $\alpha_{2}=1$, then the Berry phase associated with the loop enclosing half of the $\mathrm{BZ}$ on the $k_{i}=0$ slice is $\pi$. Because of $C_{2} T$ symmetry, the $z$ component of the Berry curvature vanishes for the $k_{i}=0$ slice, and the $\pi$-Berry phase implies the existence of a $1 \bmod 2$ band crossing in each half of the $k_{i}=0$ slice. There are, hence, $2 \bmod 4$ Weyl points on the $k_{i}=0$ plane if $\alpha_{2}=1$. For group 3, the minimal number is two, but for groups 168, 171, and 172, the minimal number is six due to the sixfold rotation. In the configuration having the minimal number of Weyl points, the Weyl points on the $k_{i}=0$ plane are related to each other by twofold or sixfold rotations, thus having the same monopole charge. Using the absence of a band crossing along high-symmetry lines, the rotation eigenvalues are the same for the $k_{i}=0$ and the $k_{i}=\pi$ slices, so that $\alpha_{2}=1$ also implies that, on the $k_{i}=\pi$ slice, there are $2 \bmod 4$ Weyl points in group 3 and 6 mod 12 Weyl points in groups 168,171 , and 172. In the configuration with the minimal number of Weyl points, those Weyl points are related by rotation symmetries thus having the same monopole charge. However, since the total charge must vanish in the entire BZ, the Weyl points on the $k_{i}=0$ and the $k_{i}=\pi$ slices have opposite charges.

Space groups 75 and 77 have a single fourfold rotation and screw axis, respectively. According to the lemma in Eq. (24), we can define on the $k_{z}=0$ slice the following $\mathbb{Z}_{2}$ indicator:

$\beta_{2} \equiv N_{\xi=-1}(\Gamma)+N_{\xi=-1}(M)+N_{\zeta=-1}(X) \bmod 2$.

The physical meaning of $\beta_{2}=1$ is that the Berry phase of the loop enclosing one quadrant of the $\mathrm{BZ}$ on the $k_{z}=0$ slice is $\pi$ and implies the presence of a Weyl point within the quadrant. Following similar steps, we find that if $\beta_{2}=1$, there are, minimally, four Weyl points on the $k_{z}=0$ plane and four on the $k_{z}=\pi$ plane. The four Weyl points having the same $k_{z}$ have the same charge, while those on different $k_{z}$ slices have opposite monopole charges.

Here, we take space group 3 as an example to show how the Weyl points can be created or, equivalently, annihilated. The minimal configuration of Weyl points is shown in Fig. 5(a). In the following, we present a process annihilating these Weyl points symmetrically. First, the two Weyl points in the $k_{y}=0$ slice move toward $\Gamma$ and then meet each other at $\Gamma$. (For space group 3, the $C_{2}$-invariant line $\Gamma Z$ is along the $k_{y}$ direction.) After their meeting, the $k_{y}=0$ slice become fully gapped. According to the lemma [Eq. (8)], this process changes the parity of the sum of $C_{2}$ eigenvalues in the occupied bands, leading to a change of $\alpha_{2}$. The interchange of $C_{2}$ eigenvalues also breaks the compatibility relation along $\Gamma Z$, leading to a band crossing protected by $C_{2}$. Second, to remove the band crossing and recover the compatibility relation, we move the crossing point to $Z$, causing a band inversion there, which changes $C_{2}$ eigenvalues at $Z$. Again, due to the lemma [Eq. (8)], this band inversion creates two additional Weyl points in the $k_{y}=\pi$ slice, possessing opposite charges of the two Weyl points that have been in $k_{y}=\pi$. Third, we annihilate the four Weyl points pairwise at two $C_{2}$-related generic momenta.

\section{B. Rotation or screw axis plus vertical glide planes (groups 27, 37, 103, and 184)}

In this subsection, the space groups all have, aside from a rotation axis, vertical glide planes whose half-translation directions are along the rotation axis, denoted by $G_{a}:(x, y, z) \rightarrow(-x, y, z+1 / 2)$. To find the expressions of the indicators, we have to invoke new variations of the lemma in Eq. (8). There are three variations for this type of space group, in simple orthorhombic, base-centered orthorhombic, and simple tetragonal Bravais lattices, respectively. (Here, we consider the hexagonal lattice of group 184 a special case of base-centered orthorhombic.) We start 

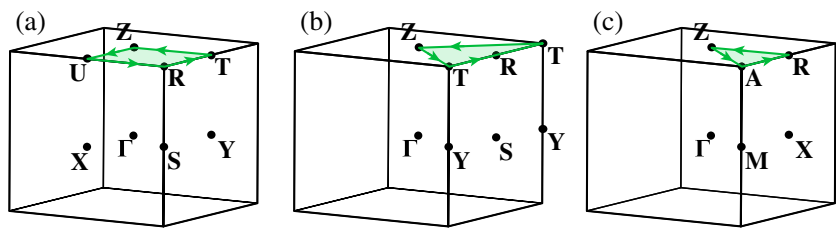

FIG. 4. Loops along which the Berry phases are used to define indicators in noncentrosymmetric space groups (a) 27, (b) 37, and (c) 103 (see Table III).

our discussion with the simple orthorhombic case, whose BZ is depicted in Fig. 4(a).

On the $k_{z}=\pi$ slice, we have $G_{a}^{2}=e^{-i k_{z}}=-1$, so that its eigenvalues $g_{a}= \pm i . C_{2}$ and $G_{a}$ automatically imply another glide plane $G_{b} \equiv C_{2} G_{a}$, which also satisfies $G_{b}^{2}=e^{-i k_{z}}=-1$ and $g_{b}= \pm i$. The two lines $Z T$ and $U R$ are invariant under $G_{a}$, while $Z U$ and $T R$ are invariant under $G_{b}$. Therefore, along these lines, bands can be labeled by their respective eigenvalues, $\left|u_{a / b, \pm i}(\mathbf{k})\right\rangle$. In fact, since $C_{2} T$ commutes with $G_{a}$ and $G_{b}$, all bands along these lines are doubly degenerate, as $C_{2} T$ maps an eigenstate having $g_{a, b}= \pm i$ to one having $g_{a, b}=\mp i$, leaving $\mathbf{k}$ unchanged on the $k_{z}=\pi$ slice. Without additional symmetries, the bands along these lines appear in groups of two. For simplicity, we assume for now that there are only two bands (one group).

The four TRIMs are not only both $G_{a, b}$ invariant but also $C_{2}$ invariant. Since $C_{2}$ eigenvalues, $\zeta= \pm 1$, are real, they are unchanged under $C_{2} T$; at each TRIM, the doublet states have the same $\zeta$. Another important fact is that, due to $G_{b}=C_{2} G_{a}$, at each TRIM we have

$$
G_{b}\left|u_{a, \pm i}(\mathbf{K})\right\rangle= \pm \zeta(\mathbf{K})\left|u_{a, \pm i}(\mathbf{K})\right\rangle
$$

which implies

$$
\left|u_{b, \pm i}(\mathbf{K})\right\rangle \propto\left|u_{a, \pm \zeta(\mathbf{K}) i}(\mathbf{K})\right\rangle .
$$

Now, we consider the Berry phase associated with the loop shown in Fig. 4(a), enclosing a quarter of the BZ at $k_{z}=\pi$. We pick a basis at $Z$ :

$$
\begin{aligned}
\left|u_{1}(Z)\right\rangle & \equiv\left|u_{a,+i}(\mathbb{Z})\right\rangle, \\
\left|u_{2}(Z)\right\rangle & =\left[\left|u_{1}(Z)\right\rangle\right]^{*} .
\end{aligned}
$$

The first segment of the path is $Z T$; along this line, the Hamiltonian can be block-diagonalized into $H_{a,+i} \oplus H_{a,-i}$. $\left|u_{1}\right\rangle$ hence evolves under $H_{a,+i}$, and $\left|u_{2}\right\rangle$ evolves under $H_{a,-i}$ such that, at $T$, they are still eigenvectors of $G_{a}$, and, since $H_{+i}=H_{-i}^{*}$ thanks to $C_{2} T$ symmetry, they still satisfy $\left|u_{2}\right\rangle=\left(\left|u_{1}\right\rangle\right)^{*}$. Using Eq. (39), we know that $\left|u_{1,2}(T)\right\rangle$ are also eigenvectors of $G_{b}$ with eigenvalues $\pm \zeta(T)$. Starting from $T$ to $R$, the two states evolve under $H_{b,+\zeta(T) i}$ and $H_{b,-\zeta(T) i}$, respectively. Repeating this process, when the two states $\left|u_{1,2}\right\rangle$ go back to $Z$, they become $\left|u_{1}^{\prime}(Z)\right\rangle$ and $\left|u_{2}^{\prime}(Z)\right\rangle$, which are eigenvectors of $G_{b}$, because the final segment of the path $U Z$ is invariant under $G_{b}$. Their eigenvalues are given by $g_{b}= \pm \zeta(T) \zeta(R) \zeta(U) i$, respectively. Using Eq. (39) at $\mathbf{K}=Z$, we find that $\left|u_{1,2}^{\prime}\right\rangle$ are also eigenvectors of $G_{a}$ :

$$
\begin{aligned}
\left|u_{1}^{\prime}(Z)\right\rangle & =e^{i \theta}\left|u_{a, \zeta(T) \zeta(R) \zeta(U) \zeta(Z) i}\right\rangle, \\
\left|u_{2}^{\prime}(Z)\right\rangle & =\left[\left|u_{1}^{\prime}(Z)\right\rangle\right]^{*} .
\end{aligned}
$$

Equations (42), hence, call for the definition of the new $\mathbb{Z}_{2}$ indicator

$$
\gamma_{2} \equiv \sum_{\mathbf{K} \in Z, T, U, R} N_{\zeta=-1}(\mathbf{K}) / 2 \bmod 2
$$

where the division by 2 is because each level is doubly degenerate. When $\gamma_{2}=1$, we have

$$
\left(\begin{array}{l}
\left|u_{1}^{\prime}\right\rangle \\
\left|u_{2}^{\prime}\right\rangle
\end{array}\right)=\left(\begin{array}{cc}
0 & e^{i \theta} \\
e^{-i \theta} & 0
\end{array}\right)\left(\begin{array}{l}
\left|u_{1}\right\rangle \\
\left|u_{2}\right\rangle
\end{array}\right)
$$

so that the Berry phase of the loop is

$$
e^{i \Phi_{B}}=\operatorname{det}\left(\left\langle u_{i}^{\prime} \mid u_{j}\right\rangle\right)=-1 .
$$

Here, we have established yet another variation of the lemma.

Lemma: Third variation.-

$$
e^{i \Phi_{B}}=\prod_{n \in \mathrm{occ} / 2, \mathbf{K} \in Z, T, U, R} \zeta_{n}(\mathbf{K})=e^{i \gamma_{2} \pi},
$$

where occ/2 means that, for each degenerate pair at these TRIMs, we take only one band for the calculation. Therefore, when $\gamma_{2}=1$, the Berry phase of the loop in Fig. 4(a) is $\pi$, and, since $C_{2} T$ ensures the vanishing of the $z$ component of the Berry curvature, there must be $1 \bmod 2$ Weyl point in each quadrant. In the configuration having the least number (four) of Weyl points, the two Weyl points related by $G_{a}$ or $G_{b}$ have opposite monopole charges.

It may be a little counterintuitive why the two Weyl points of opposite charge cannot pairwise annihilate on the glide plane. Consider two such Weyl points that are related to each other by $G_{a}$. Then, we remark that they are necessarily also related by $G_{b} * T$ and that, on the $k_{z}=\pi$ slice, there is

$$
\left(\hat{G}_{b} \hat{T}\right)^{2}=\hat{G}_{b}^{2} \hat{T}^{2}=-1 .
$$

In Ref. [17], one of us shows that two Weyl points related by $G_{b} * T$ satisfying $\left(\hat{G}_{b} \hat{T}\right)^{2}=-1$ cannot pairwise annihilate when they meet but will form an accidental Dirac point. 
The discussion of a base-centered orthorhombic case differs but a little from the simple orthorhombic case. Again, we consider the $k_{z}=\pi$ slice, where the TRIMs are denoted $Z, R, T$, whose multiplicities are one, two, and one, respectively. While all TRIMs are $C_{2}$ invariant, $Z$ and $T$ are also invariant under $G_{a}$ and $G_{b}$. Consider the loop shown in Fig. 4(b). One should notice that, while the loop encloses one-quarter of the BZ, it is formed by two closed loops, labeled loop 1 from $T$ to $R$ to $T$ and loop 2 from $T$ to $Z$ to $T$. The total Berry phase is the sum of the Berry phases of loop 1 and loop 2, each of which is quantized to 0 and $\pi$ due to $C_{2} T$. Using the same steps, we can show that the second Berry phase is given by

$$
\exp \left(i \Phi_{2}\right)=\prod_{n \in \mathrm{occ} / 2} \zeta_{n}(Z) \zeta_{n}(T)
$$

where occ/2 means that for each degenerate pair only one state is chosen. Regarding loop 1, Refs. [37,38] show that its Berry phase is given by

$$
\exp \left(i \Phi_{1}\right)=\prod_{n \in \mathrm{occ}} \zeta_{n}(T) \zeta_{n}(R)=\prod_{n \in \mathrm{occ}} \zeta_{n}(R)
$$

where the second equality comes from the fact that all $\zeta$ values at $T$ appear in doubles. These observations lead us to define a new $\mathbb{Z}_{2}$ indicator:

$$
\gamma_{2}^{\prime} \equiv \frac{N_{\zeta=-1}(Z)+N_{\zeta=-1}(T)}{2}+N_{\zeta=-1}(R) \bmod 2
$$

such that $e^{i\left(\Phi_{1}+\Phi_{2}\right)}=e^{i \gamma_{2}^{\prime} \pi}$. When $\gamma_{2}^{\prime}=1$, there must be one Weyl point inside each quarter of the $\mathrm{BZ}$ at the $k_{z}=\pi$ slice. Therefore, there are four Weyl points in the minimal configuration, where any two related by either $G_{a}$ or $G_{b}$ have opposite monopole charges.

The indicator $\gamma_{2}^{\prime}$ is well defined for both groups 37 and 183 , since the hexagonal lattice can be considered as a special case of a base-centered orthogonal lattice. However, in group 183, due to the sixfold rotation, the total number of Weyl points is $12 \bmod 24$, and in the minimal configuration, 12 Weyl points are related to each other either by $G_{a}$ or by $C_{6}$, shown in Fig. 5(h). Two Weyl points related by $C_{6}$ have the same monopole charge, and two related by $G_{a, b}$ have opposite charges.

Similarly, we find the indicator for space group 103:

$\gamma_{2}^{\prime \prime} \equiv \frac{N_{\xi=-1}(Z)+N_{\xi=-1}(A)+N_{\zeta=-1}(R)}{2} \bmod 2$.

When $\gamma_{2}^{\prime \prime}=1$, the Berry phase of the loop in Fig. 4(c) is $\pi$, so that there must be $1 \bmod 2$ Weyl point in half of a quadrant, resulting in $8 \bmod 16$ Weyl points in total. In the minimal configuration, the eight Weyl points, four positive and four negative, are related to each other by either $C_{4}$ or $G_{a, b}$. Two Weyl points related by $C_{4}$ have the same monopole charge, and the two related by $G_{a, b}$ have different ones.

\section{C. $S_{4}$ symmetry (groups 81 and 82)}

$S_{2 n}$ symmetries are the only type of (noncentrosymmetric) point group symmetries the invariant subspace of which consists of discrete points rather than lines or planes. Aside from $S_{2}$, which is same as inversion, $S_{4}$ is also the only $S_{2 n}$ symmetry that is consistent with 3D lattices. Space group 81 on a simple tetragonal lattice is singly generated by this symmetry. In momentum space, we have $S_{4}:\left(k_{x}, k_{y}, k_{z}\right) \rightarrow\left(-k_{y}, k_{z},-k_{z}\right)$, so that on $k_{z}=0$ and $k_{z}=\pi$ slices, they act the same way as $C_{4}$. Therefore, we can use the same lemma in Eq. (24) to define the following two $\mathbb{Z}_{2}$ indicators:

$\omega_{2}^{0}=N_{\xi=-1}(\Gamma)+N_{\xi=-1}(M)+N_{\zeta=-1}(X) \bmod 2$,

$\omega_{2}^{\pi}=N_{\xi=-1}(Z)+N_{\xi=-1}(A)+N_{\zeta=-1}(R) \bmod 2$,

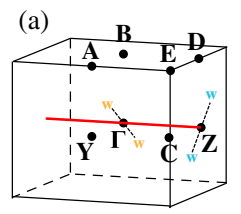

(b)

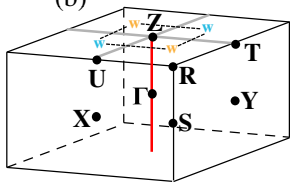

(g)

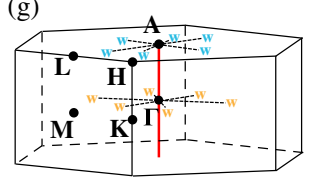

(h)

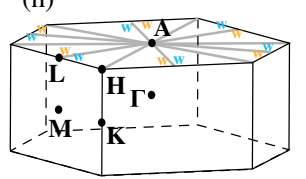

(c)

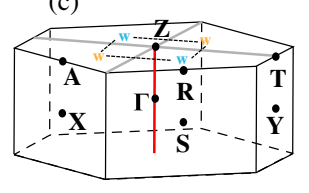

(i)

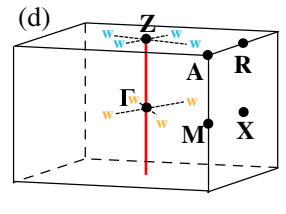

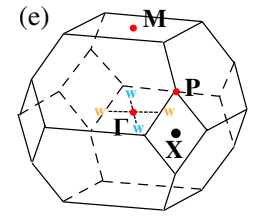

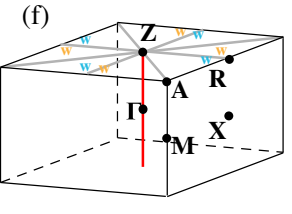

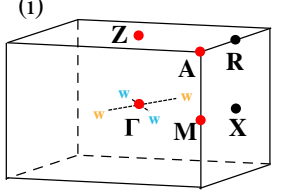
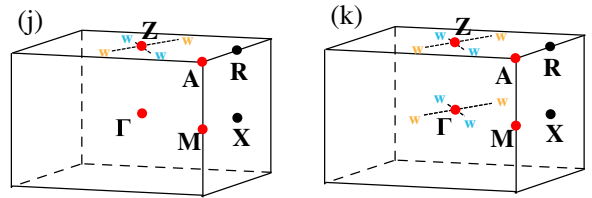

FIG. 5. Minimal configurations of the Weyl points for each nonzero set of indicators in various space groups. Red lines are rotation axes, gray lines the intersection between vertical glide planes and the $k_{z}=\pi$ plane, red dots $S_{4}$ centers, and " $w$ " stands for Weyl, where different colors of $w$ mean opposite monopole charge. (a)-(h) are for space groups with only one $\mathbb{Z}_{2}$ indicator: (a) 3, (b) 27, (c) 37, (d) 75 and 77, (e) 82, (f) 103, (g) 168, 171, and 172, and (h) 184. (i)-(k) are the minimal configurations for group 81 having indicator sets $\left(\omega_{2}^{0}, \omega_{2}^{\pi}\right)=(1,0),(0,1),(1,1)$, respectively. 
where $\xi$ is the $S_{4}$ eigenvalue and we observed the fact that $S_{4}^{2}=C_{2}$.

When $\omega_{2}^{0, \pi}=1$, there are $4 \bmod 8$ points on the $k_{z}=0$ and the $k_{z}=\pi$ slice, respectively. Since $S_{4}$ is an improper rotation, two Weyl points related by $S_{4}$ have opposite monopole charges: This result is a difference between $S_{4}$ and $C_{4}$.

For space group 82 , the only $\mathbb{Z}_{2}$ indicator is $\omega_{2}^{0}$.

\section{DISCUSSION AND CONCLUSION}

In this work, we find the explicit expressions for all symmetry-based indicators in terms of symmetry eigenvalues of valence bands at high-symmetry points in the momentum space for 3D systems with time-reversal symmetry and negligible spin-orbital coupling, and we characterize all topological states corresponding to every given set of nonzero indicators. Somewhat to our surprise, all nonzero sets of indicators necessarily correspond to some topological semimetals; i.e., none of them is compatible with a gapped band structure in three dimensions. These semimetals are, in general, "hidden" from most firstprinciples calculations, since in the latter only highsymmetry lines are routinely scanned, but in these semimetals all high-symmetry lines have gapped spectra. Looking at these expressions, we realize that all the indicators except one are, in fact, topological invariants for submanifolds of the 3D BZ. (These expressions are checked via decomposing all linearly independent solutions of compatibility relations into elementary band representations, where both data can be found in Ref. [26].)

Most of the indicators are equivalent to Berry phases:

(i) $z_{2, i=1,2,3} \pi$ is the Berry phase of a loop enclosing half of the $\mathrm{BZ}$ at $k_{i}=\pi$ (all centrosymmetric space groups).

(ii) $z_{2}^{( \pm)} \pi$ is the Berry phase of a loop enclosing half of the $\mathrm{BZ}$ at $k_{z}=\pi$ in the $M= \pm 1$ sector (groups 10, 175,176 , and 192).

(iii) $\delta_{2} \pi$ is the Berry phase of a loop enclosing a quarter of the BZ at any fixed $k_{z}$ (groups $83,84,85$, and 86).

(iv) $\delta_{2}^{( \pm), \pi} \pi$ is the Berry phase of a loop enclosing a quarter of the $\mathrm{BZ}$ at $k_{z}=\pi$ in the $M= \pm 1$ sector (groups 83, 124, and 128).

(v) $\delta_{2}^{( \pm), 0} \pi$ is the Berry phase of a loop enclosing a quarter of the BZ at $k_{z}=0$ in the $M= \pm 1$ sector (groups 83, 84, and 87).

(vi) $\delta_{2}^{\prime} \pi$ is the difference between the Berry phase of a loop enclosing a quarter of the $\mathrm{BZ}$ at $k_{z}=0$ in the $M=+1$ sector and that of a loop and that at $k_{z}=\pi$ (group 83).

(vii) $\phi_{2} \pi$ is the Berry phase of the loop indicated in Fig. 3 (group 87).

(viii) $\theta_{2} \pi$ is conjectured to be the Berry phase of the loop indicated in Fig. 1(c) (group 130). (ix) $\alpha_{2} \pi$ is the Berry phase of a loop enclosing half of the $\mathrm{BZ}$ at $k_{z}=0$ (groups 3,168, 171, and 172).

(x) $\beta_{2} \pi$ is the Berry phase of a loop enclosing a quarter of the $\mathrm{BZ}$ at $k_{z}=0$ (groups 75 and 77).

(xi) $\gamma_{2} \pi$ is the Berry phase of a loop enclosing a quarter of the $\mathrm{BZ}$ at $k_{z}=\pi$ (group 27).

(xii) $\gamma_{2}^{\prime} \pi$ is the Berry phase of a loop enclosing quarter of $\mathrm{BZ}$ at $k_{z}=\pi(\# 37,184)$.

(xiii) $\gamma_{2}^{\prime \prime} \pi$ is the Berry phase of a loop enclosing one-eighth of the $\mathrm{BZ}$ at $k_{z}=\pi$ (group 103).

(xiv) $\omega_{2}^{0} \pi$ is the Berry phase of a loop enclosing a quarter of the BZ at the $k_{z}=0$ plane (groups 81 and 82).

(xv) $\omega_{2}^{\pi} \pi$ is the Berry phase of a loop enclosing a quarter of the $\mathrm{BZ}$ at the $k_{z}=\pi$ plane (group 81).

There is one special $\mathbb{Z}_{4}$ indicator, $z_{4}$, that is not a Berry phase but the number of nodal rings modulo four; $z_{2}^{\prime}=$ $z_{4} / 2$ is a derivative of $z_{4}$ in space groups where $z_{4}$ is constrained by symmetries to be even.

Whenever any of the above Berry phases is $\pi$, we immediately know that there are 1 mod 2 robust band crossings in the area bounded by the loop. Whenever $z_{4}$ or $z_{2}^{\prime}$ is nonzero, it gives us the total number of rings or lines. These observations help us determine all possible configurations of all the nodal loops and Weyl points given any set of symmetry-based indicators mentioned above.

Importantly, the definitions of these indicators in most cases require only several, but not all, symmetries in the space groups in which they are defined, and they require only that there is no band crossing along the loop on which the Berry phase is defined, which means that, even in band structures where compatibility relations are violated, i.e., when there are band crossings along certain high-symmetry lines, as long as the above requirements hold, these indicators can still be applied to find band crossings at generic momenta, away from the ones on high-symmetry lines.

A very simple example helps illustrate this point. In space group 75, which has a simple tetragonal lattice and a single point-group generator $C_{4}$, starting from a gapped state, we consider a band inversion at $\Gamma$ where the valence band with $\xi=+1$ becomes one having $\xi=-1$. This band inversion is known to cause two band crossings along $\Gamma Z$, since $C_{4}$ is a good quantum number along this line, so the band structure violates the compatibility relations of group 75 . However, one easily confirms that $\beta_{2}=1$ after the band inversion, so that, away from $\Gamma Z$, there are four additional Weyl points on the $k_{z}=0$ plane, having the same monopole charge.

Topological semimetals are known to be "parent states" for topologically gapped states. In fact, two possible mechanisms have been established that give rise to nontrivial topology: the Kane-Mele mechanism [19,20], where topological band crossings open up a gap when spin-orbital coupling is turned on, and the Bernevig-Hughes-Zhang 
mechanism [39], where band inversions occur due to strong spin-orbital coupling. These two mechanisms (and their generalized versions) are used and so far considered as guiding principles for the search of topological materials that are systematically applied in Ref. [26], which have successfully yielded many a new material candidate. (Among them are new types of "weak phases" termed fragile topological phases, the understanding of which opens new directions [40,41].) According to the KaneMele mechanism, some topological semimetals, like graphene, are just topological (crystalline) insulators disguised by the small SOC. A question then naturally presents itself at this point: Will the topological semimetals diagnosed by these indicators become topological gapped states, such as topological insulators or topological crystalline insulators [42], when spin-orbital coupling is perturbatively added? For noncentrosymmetric space groups, the answer is very simple: Each Weyl points splits into two having the same charge, so that the system remains a Weyl semimetal. For centrosymmetric space groups, since neither a Weyl point or nodal line at generic momenta is allowed, adding SOC will open full gaps for all topological semimetals discussed in this work. But are all these gapped states topologically nontrivial? A complete answer to this question justifies an independent work, but here we partly address this issue by connecting the indicators in the orthogonal (without SOC) and the symplectic (with SOC) classes. The topological information carried by the nonzero indicators in the symplectic class is discussed in a parallel work [43,44].

To be specific, we inspect all space groups that have a nontrivial indicator group both with and without SOC. We find the following quantitative relations between the indicators in the two classes of Hamiltonians. First of all, the indicators that depend only on inversion eigenvalues $z_{2, i=1,2,3}$ and $z_{4}$ have exactly the same definition in both classes. The Fu-Kane formula shows that, with SOC, $z_{2, i=1,2,3}$ and $z_{4} \bmod 2$ correspond to the three weak and one strong invariants of time-reversal topological insulators. When $z_{2, i}=0$ and $z_{4}=2$, the corresponding gapped states with SOC belong to a new class of topological crystalline insulators, the surface states of which are discussed in Refs. [43-45]. Among centrosymmetric space groups having nontrivial indicator groups both with and without SOC, these four indicators have comprehended the indicator groups in both classes in all but the following five space groups: 83, 87, 128, 175, and 192 .

To find the relations between indicators without SOC and those with SOC, we first need to determine what an irreducible representation without SOC becomes when SOC is turned on. To be specific, if a irreducible representation without SOC at $\mathbf{k}$ is given by $D(g)$, where $g$ belongs to the little group of $\mathbf{k}$, then the representation matrix after considering an infinite small SOC becomes $D(g) \otimes u(g)$, where $u(g)$ is the $\mathrm{SU}(2)$ rotation matrix of $g$.
In most cases, $D(g) \otimes u(g)$ becomes reducible and reduces to several irreducible representations with SOC. With this information obtained, we are able to translate any symmetry data without SOC to symmetry data with SOC. Following this method, we find the following.

(i) Group 83.-The indicators in the absence of SOC are $\delta_{2}^{(+), \pi}, \delta_{2}^{(-), \pi}$, and $\delta_{2}^{\prime}$. The indicators in the presence of SOC are $z_{2 w, 1}, z_{4 m, \pi}$, and $z_{8}$, wherein $z_{2 w, 1}$ is the weak index in the $x$ direction, $z_{4 m, \pi}$ is the mirror Chern number $(\bmod 4)$ at the $k_{z}=\pi$ plane, and $z_{8} \bmod 4$ is the sum of mirror Chern numbers at the $k_{z}=0$ and $k_{z}=\pi$ planes $(\bmod 4)$. The remaining case, $z_{8}=4$, corresponds to either a $C_{4}$-protected topological crystalline insulator or a mirror Chern insulator with mirror Chern numbers at the $k_{z}=0$ - and $k_{z}=\pi$ planes differing from each other by $4(\bmod 8)$ [43-46]. The mappings from indicators without SOC to indicators with SOC are $(100) \rightarrow(024)$, $(010) \rightarrow(024)$, and $(001) \rightarrow(004)$.

(ii) Group 87.- The indicators in the absence of SOC are $\phi_{2}$ and $\delta_{2}^{(+), 0}$, and the indicators in the presence of SOC are $z_{2 w, 1}$ and $z_{8}$, which have the same definitions in group $83[43,44]$. The mappings are $(10) \rightarrow(04)$ and $(01) \rightarrow(04)$.

(iii) Group 128.-The indicator in the absence of SOC is $\delta_{2}^{(+), \pi}$, and the indicator in the presence of SOC is $z_{8}$, which has the same definition in group 83 [43,44]. The mapping is $1 \rightarrow 4$.

(iv) Group 175.- The indicators in the absence of SOC are $z_{2}^{(+)}, z_{2}^{(-)}$, and $z_{2}^{\prime}=z_{4} / 2$. The indicators in the presence of SOC are $z_{6 m, \pi}$ and $z_{12}$, wherein $z_{6 m, \pi}$ is the mirror Chern number $(\bmod 6)$ at the $k_{z}=\pi$ plane and $z_{12}$ mod 6 is the sum of mirror Chern numbers at the $k_{z}=0$ and $k_{z}=\pi$ planes. The remaining case, $z_{12}=6$, corresponds to either a $C_{6}$-protected topological crystalline insulator or a mirror Chern insulator with mirror Chern numbers at the $k_{z}=0$ and $k_{z}=\pi$ planes differing from each other by 6 $(\bmod 12)[43,44]$. The mappings are $(100) \rightarrow(30)$, $(010) \rightarrow(30)$, and $(001) \rightarrow(06)$.

(v) Group 192.- The indicator in the absence of SOC is $z_{2}^{\prime}$, and the indicator in the presence of SOC is $z_{12}$, which has the same definition in group 175 . The mapping is $1 \rightarrow 6$.

\section{ACKNOWLEDGMENTS}

The authors acknowledge support from Ministry of Science and Technology of China under Grants No. 2016YFA0302400 and No. 2016YFA0300600, National Science Foundation of China under Grants No. 11674370 and No. 11421092, and Chinese Academy of Sciences under Grant No. XXH13506-202. 
[1] S. Murakami, Phase Transition between the Quantum Spin Hall and Insulator Phases in 3D: Emergence of a Topological Gapless Phase, New J. Phys. 9, 356 (2007).

[2] X. Wan, A. M. Turner, A. Vishwanath, and S. Y. Savrasov, Topological Semimetal and Fermi-Arc Surface States in the Electronic Structure of Pyrochlore Iridates, Phys. Rev. B 83, 205101 (2011).

[3] A. A. Burkov, Topological Semimetals, Nat. Mater. 15, 1145 (2016).

[4] A. A. Burkov, M. D. Hook, and L. Balents, Topological Nodal Semimetals, Phys. Rev. B 84, 235126 (2011).

[5] L. Lu, L. Fu, J. D. Joannopoulos, and M. Soljacic, Weyl Points and Line Nodes in Gyroid Photonic Crystals, Nat. Photonics 7, 294 (2013).

[6] L. Lu, Z. Wang, D. Ye, L. Ran, L. Fu, J. D. Joannopoulos, and M. Soljacic, Experimental Observation of Weyl Points, Science 349, 622 (2015).

[7] O. Stenull, C. L. Kane, and T. C. Lubensky, Topological Phonons and Weyl Lines in Three Dimensions, Phys. Rev. Lett. 117, 068001 (2016).

[8] T. Zhang, Z. Song, A. Alexandradinata, H. Weng, C. Fang, L. Lu, and Z. Fang, Double-Weyl Phonons in TransitionMetal Monosilicides, Phys. Rev. Lett. 120, 016401 (2018).

[9] F.-Y. Li, Y.-D. Li, Y. B. Kim, L. Balents, Y. Yu, and G. Chen, Weyl Magnons in Breathing Pyrochlore Antiferromagnets, Nat. Commun. 7, 12691 (2016).

[10] J. Fransson, A. M. Black-Schaffer, and A. V. Balatsky, Magnon Dirac Materials, Phys. Rev. B 94, 075401 (2016).

[11] K. Li, C. Li, J. Hu, Y. Li, and C. Fang, Dirac and Nodal Line Magnons in Three-Dimensional Antiferromagnets, Phys. Rev. Lett. 119, 247202 (2017)..

[12] W. Yao, C. Li, L. Wang, S. Xue, Y. Dan, K. Iida, K. Kamazawa, K. Li, C. Fang, and Y. Li, Topological Spin Excitations Observed in a Three-Dimensional Antiferromagnet, arXiv:1711.00632.

[13] S. Bao, J. Wang, W. Wang, Z. Cai, S. Li, Z. Ma, D. Wang, K. Ran, Z.-Y. Dong, D. L. Abernathy, X. Wan, S.-L. Yu, J.-X. Li, and J. Wen, Observation of Dirac Magnons in a ThreeDimensional Antiferromagnet $\mathrm{Cu}_{3} \mathrm{TeO}_{6}$, arXiv:1711.02960.

[14] B. Q. Lv, H. M. Weng, B. B. Fu, X. P. Wang, H. Miao, J. Ma, P. Richard, X. C. Huang, L. X. Zhao, G. F. Chen, Z. Fang, X. Dai, T. Qian, and H. Ding, Experimental Discovery of Weyl Semimetal TaAs, Phys. Rev. X 5, 031013 (2015).

[15] S.-Y. Xu, I. Belopolski, N. Alidoust, M. Neupane, C. Zhang, R. Sankar, S.-M. Huang, C.-C. Lee, G. Chang, B. Wang, G. Bian, H. Zheng, D. S. Sanchez, F. Chou, H. Lin, S. Jia, and M. Zahid Hasan, Experimental Realization of a Topological Weyl Semimetal Phase with Fermi Arc Surface States in TaAs, Science 349, 613 (2015).

[16] G. Bian, T.-R. Chang, R. Sankar, S.-Y. Xu, H. Zheng, T. Neupert, C.-K. Chiu, S.-M. Huang, G. Chang, I. Belopolski, D. S. Sanchez, M. Neupane, N. Alidoust, C. Liu, B. Wang, C.-C. Lee, H.-T. Jeng, C. Zhang, Z. Yuan, S. Jia et al., Topological Nodal-Line Fermions in Spin-Orbit Metal $\mathrm{PbTaSe}_{2}$, Nat. Commun. 7, 10556 (2016).

[17] C. Fang, L. Lu, J. Liu, and L. Fu, Topological Semimetals with Helicoid Surface States, Nat. Phys. 12, 936 (2016).

[18] D. T. Son and B. Z. Spivak, Chiral Anomaly and Classical Negative Magnetoresistance of Weyl Metals, Phys. Rev. B 88, 104412 (2013).
[19] C. L. Kane and E. J. Mele, Quantum Spin Hall Effect in Graphene, Phys. Rev. Lett. 95, 226801 (2005).

[20] C. L. Kane and E. J. Mele, $Z_{2}$, Phys. Rev. Lett. 95, 146802 (2005).

[21] S.-M. Huang et al., An Inversion Breaking Weyl Semimetal State in the TaAs Material Class, Nat. Commun. 6, 7373 (2015).

[22] H. Weng, C. Fang, Z. Fang, B. A. Bernevig, and X. Dai, Weyl Semimetal Phase in Noncentrosymmetric TransitionMetal Monophosphides, Phys. Rev. X 5, 011029 (2015).

[23] G. Kresse and J. Furthmuller, Efficiency of Ab-Initio Total Energy Calculations for Metals and Semiconductors Using a Plane-Wave Basis Set, Comput. Mater. Sci. 6, 15 (1996).

[24] R. Yu, X. L. Qi, A. Bernevig, Z. Fang, and X. Dai, Equivalent Expression of $\boldsymbol{F}_{2}$ Topological Invariant for Band Insulators Using the Non-Abelian Berry Connection, Phys. Rev. B 84, 075119 (2011).

[25] H. C. Po, A. Vishwanath, and H. Watanabe, SymmetryBased Indicators of Band Topology in the 230 Space Groups, Nat. Commun. 8, 50 (2017).

[26] B. Bradlyn, L. Elcoro, J. Cano, M. G. Vergniory, Z. Wang, C. Felser, M. I. Aroyo, and B. A. Bernevig, Topological Quantum Chemistry, Nature (London) 547, 298 (2017).

[27] J. Kruthoff, J. de Boer, J. van Wezel, C. L. Kane, and R.-J. Slager, Topological Classification of Crystalline Insulators through Band Structure Combinatorics, Phys. Rev. X 7, 041069 (2017)..

[28] M. G. Vergniory, L. Elcoro, Z. Wang, J. Cano, C. Felser, M. I. Aroyo, B. A. Bernevig, and B. Bradlyn, Graph Theory Data for Topological Quantum Chemistry, Phys. Rev. E 96, 023310 (2017).

[29] L. Elcoro, B. Bradlyn, Z. Wang, M. G. Vergniory, J. Cano, C. Felser, B. A. Bernevig, D. Orobengoa, G. de la Flor, and Mois I. Aroyo, Double Crystallographic Groups and Their Representations on the Bilbao Crystallographic Server, J. Appl. Crystallogr. 50, 1457 (2017).

[30] M. I. Aroyo, J. M. Perez-Mato, C. Capillas, E. Kroumova, S. Ivantchev, G. Madariaga, A. Kirov, and H. Wondratschek, Bilbao Crystallographic Server: I. Databases and Crystallographic Computing Programs, Z. Kristallogr. 221, 15 (2009).

[31] M. I. Aroyo, D. Orobengoa, G. de la Flor, E. S. Tasci, J. M. Perez-Mato, and H. Wondratschek, Brillouin-Zone Database on the Bilbao Crystallographic Server, Acta Crystallogr. Sect. A 70, 126 (2014).

[32] E. P. Wigner, Characteristic Vectors of Bordered Matrices with Infinite Dimensions, Ann. Math. 62, 548 (1955).

[33] A. Altland and M. R. Zirnbauer, Nonstandard Symmetry Classes in Mesoscopic Normal-Superconducting Hybrid Structures, Phys. Rev. B 55, 1142 (1997).

[34] Y. Kim, B. J. Wieder, C. L. Kane, and A. M. Rappe, Dirac Line Nodes in Inversion-Symmetric Crystals, Phys. Rev. Lett. 115, 036806 (2015).

[35] C. Fang, Y. Chen, H.-Y. Kee, and L. Fu, Topological Nodal Line Semimetals with and without Spin-Orbital Coupling, Phys. Rev. B 92, 081201 (2015).

[36] C. Fang, M. J. Gilbert, and B. A. Bernevig, Bulk Topological Invariants in Noninteracting Point Group Symmetric Insulators, Phys. Rev. B 86, 115112 (2012). 
[37] A. M. Turner, Y. Zhang, and A. Vishwanath, Entanglement and Inversion Symmetry in Topological Insulators, Phys. Rev. B 82, 241102 (2010).

[38] T. L. Hughes, E. Prodan, and B. A. Bernevig, InversionSymmetric Topological Insulators, Phys. Rev. B 83, 245132 (2011).

[39] B. A. Bernevig, T. L. Hughes, and S.-C. Zhang, Quantum Spin Hall Effect and Topological Phase Transition in HgTe Quantum Wells, Science 314, 1757 (2006).

[40] H. C. Po, H. Watanabe, and A. Vishwanath, Fragile Topology and Wannier Obstructions, arXiv:1709.06551.

[41] J. Cano, B. Bradlyn, Z. Wang, L. Elcoro, M. G. Vergniory, C. Felser, M. I. Aroyo, and B. A. Bernevig, Building Blocks of Topological Quantum Chemistry: Elementary Band Representations, Phys. Rev. B 97, 035139 (2018).
[42] L. Fu, Topological Crystalline Insulators, Phys. Rev. Lett. 106, 106802 (2011).

[43] Z. Song, T. Zhang, Z. Fang, and C. Fang, Mapping Symmetry Data to Topological Invariants in Nonmagnetic Materials, arXiv:1711.11049.

[44] E. Khalaf, H. C. Po, A. Vishwanath, and H. Watanabe, following paper, Symmetry Indicators and Anomalous Surface States of Topological Crystalline Insulators, Phys. Rev. X 8, 031070 (2018).

[45] C. Fang and L. Fu, Rotation Anomaly and Topological Crystalline Insulators, arXiv:1709.01929.

[46] Z. Song, Z. Fang, and C. Fang, (d-2)-Dimensional Edge States of Rotation Symmetry Protected Topological States, Phys. Rev. Lett. 119, 246402 (2017). 